\title{
A New Flux Splitting Scheme
}

Meng-Sing Liou and Christopher J. Steffen,Jr.

Lewis Research Center

Cleveland, Ohio

May 1991

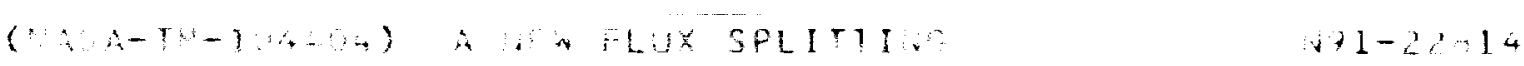

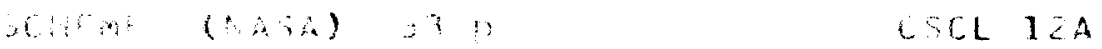

$$
\begin{aligned}
& 03104 \quad 001349
\end{aligned}
$$





\title{
A NEW FLUX SPLITTING SCHEME
}

\author{
Meng-Sing Liou* \\ and \\ Christopher J. Steffen, Jr. $†$ \\ National Aeronautics and Space Administration \\ Lewis Research Center \\ Cleveland, Ohio 44135
}

\begin{abstract}
A new flux splitting scheme is proposed. The scheme is remarkably simple and yet its accuracy rivals and in some cases surpasses that of Roe's solver in the Euler and Navier-Stokes solutions carried out in this study. The scheme is robust and converges as fast as the Roe splitting. We propose an appropriately defined cell-face advection Mach number using values from the two straddling cells via associated characteristic speeds. This interface Mach number is then used to determine the upwind extrapolation for the convective quantities. Accordingly, the name of the scheme is coined as Advection Upstream Splitting Method (AUSM). We also introduce a new pressure splitting which is shown to behave successfully, yielding much smoother results than other existing pressure splittings. Of particular interest is the supersonic blunt body problem in which the Roe scheme gives anomalous solutions. The AUSM produces correct solutions without difficulty for a wide range of flow
\end{abstract}

\footnotetext{
* Senior Scientist

$\dagger$ Aerospace Engineer
} 
conditions as well as grids.

\section{INTRODUCTION}

Maximizing both accuracy and efficiency has been the primary goal for designing an algorithm in numerical analysis. This is especially important for solution of complex 3D problems described by the Navier-Stokes equations which may include equations for a turbulence model and/or chemical species. Recently upwind schemes have become well-received for the sound theoretical basis of characteristic theory for hyperbolic systems and thus for their capability of capturing discontinuties. Furthermore, many advanced shock capturing techniques such as TVD are based on upwind schemes.

Several prominent flux splitting schemes have been compared in the literature. Liou and Van Leer [1] tested three techniques, namely the Steger-Warming (SWS), Van Leer (VLS) and Roe splittings (RS), for a variety of problems regarding their accuracy and efficiency. Osher's splitting (OS) has recently been compared with Van Leer's by Koren [2] and extended to both equilibrium and non-equilibrium chemistry by Suresh and Liou [3,4]. Flux-vector splitting (FVS) such as VLS and SWS has proved to be a simple and useful technique for arriving at upwind differencing and is pre-eminently suited for use in implicit schemes. Unfortunately, the simplicity of these two splittings comes at a price of reduced accuracy due to numerical diffusion. Flux-difference splitting (FDS) such as RS and OS, however, has shown to be very accurate and particularly well suited for explicit upwind formulations. Nevertheless, the increased accuracy is accompanied with an increased operation count and complexity in arriving at the complete linearization of flux formulas for the implicit schemes. Hence, the simplicity of FVS is still motivating researchers to investigate new ways of splitting and combinations of FVS and FDS that do away with the problem of numerical diffusion with only a small (if any) 
increase in complexity.

In SWS, glitches exist at a point where eigenvalues change sign, such as sonic or shock point. Although fixes have been proposed, it is generally agreed that the other three splittings are preferred within the framework of upwind discretization. As pointed out by Van Leer himself [5], the Van Leer splitting fails to recognize the contact discontinuity, leading to excessive numerical diffusion. As a result, significant error appears in the viscous region which can not be simply cured by reducing grid size and/or using higher-order differencing. This fact had largely gone unnoticed until 1987 when Van Leer again brought this deficiency to the attention of researchers [6]. Subsequently, several efforts have been attempted to improve the original Van Leer scheme, in particular by Hänel and Schwane et al $[7,8]$. Van Leer, recently based upon [8], has made a drastic improvement [9], in which the temperature distribution of a hypersonic conic flow is predicted as accurately as using Roe's splitting. However, a pressure glitch is accompanied with this new scheme. Another approach was suggested by Liou and Steffen [11] and Coirier and Van Leer [18] to include higher order polynomial expansions in the split fluxes in order to yield vanishing value at $M=0$. However, this is found to be less stable or oscillatory.

Hence, the current research is motivated by the desire to combine the efficiency of FVS and the accuracy of FDS. In this paper, we will present a new flux splitting scheme (details given in the next section) that is remarkably simple and accurate, yielding vanishing numerical diffusivity at the stagnation. In a variety of Euler and Navier-Stokes calculations performed, the accuracy of the present scheme is shown to be rivaling that of Roe's splitting. Also, the scheme has no matrix operation, is much simpler to construct and thus more efficient. Futhermore, unlike Roe's splitting, the scheme does not involve differentiation of fluxes, hence it is smoother. This is desirable for the general equation of state where a stiff variation of thermodynamic states may occur in certain transitional states, such as the case of chemically 
reacting flows. The scheme is a mixture, but has the advantages of both flux-vector and flux-difference splittings.

\section{ANALYSIS}

\section{Motivation:}

To exemplify the concept, let us consider the quasi two-dimensional system of equations for conical flows:

$$
\frac{\partial \mathbf{U}}{\partial t}+\frac{\partial\left(\mathbf{F}_{i n v}+\mathbf{F}_{v}\right)}{r \partial \theta}=\mathbf{S}
$$

where $\mathbf{U}^{T}=(\rho, \rho u, \rho v, \rho E)$, the inviscid flux $\mathbf{F}_{\text {inv }}^{T}=\left(\rho v, \rho v u, \rho v^{2}+p, \rho v H\right)$, and the specific total energy $E=e+1 / 2\left(u^{2}+v^{2}\right)=H-p / \rho$. The viscous flux $\mathbf{F}_{v}^{T}=-\left(0, \tau_{r \theta}, \tau_{\theta \theta}, v \tau_{\theta \theta}+u \tau_{r \theta}-q_{\theta}\right)$, with:

$$
\begin{aligned}
\tau_{\theta \theta} & =\frac{2 \mu}{3 r}\left(2 \frac{\partial v}{\partial \theta}+u-v c t\right) ; \quad c t=\cot \theta \\
\tau_{r \theta} & =\frac{\mu}{r}\left(\frac{\partial u}{\partial \theta}-v\right)
\end{aligned}
$$

and

$$
q_{\theta}=-\frac{\kappa}{r} \frac{\partial T}{\partial \theta}
$$

The source term $\mathbf{S}$ includes both inviscid and viscous contributions:

$$
\mathrm{S}=-\frac{\rho}{r}\left(\begin{array}{c}
2 u+v c t \\
u(2 u+v c t)-v^{2} \\
v(2 u+v c t)+u v \\
H(2 u+v c t)
\end{array}\right)+\frac{1}{r}\left(\begin{array}{c}
0 \\
\tau_{r r}-\tau_{\theta \theta}-\tau_{\phi \phi}+\tau_{r \theta} c t \\
2 \tau_{r \theta}+\left(\tau_{\theta \theta}-\tau_{\phi \phi}\right) c t \\
v\left(\tau_{r \theta}+\tau_{\theta \theta} c t\right)+u\left(\tau_{r r}+\tau_{r \theta} c t\right)-q_{\theta} c t
\end{array}\right)
$$

where

and

$$
\tau_{\phi \phi}=\frac{2 \mu}{3 r}\left(-\frac{\partial v}{\partial \theta}+u+2 v c t\right)
$$




$$
\tau_{r r}=-\frac{2 \mu}{3 r}\left(\frac{\partial v}{\partial \theta}+2 u+v c t\right)
$$

Since both longitudinal and transverse momentum equations are included, this system can serve as a model equation for dealing with flux terms in multidimensions. Furthermore, the supersonic conical viscous flow that consists of a very thin shear layer at the wall and a shock wave away from the wall is an ideal problem for testing the capability of capturing a shock discontinuity together with a sharp gradient boundary layer. Thus, an algorithm must be designed to minimize the numerical smearing (diffusion) at the locations where an eigenvalue changes sign or approaches zero. For example, Van Leer's splitting [5] can represent a shock profile well, while it greatly diffuses the boundary layer. It is precisely this original intent for making a smoother transition at sonic points that creates enormous diffusion at $M=0$; see Fig. 1 for the split mass distribution. The split flux deviates the most from the true flux at $M=0$. As a result of this nonvanishing flux at $M=0$, the boundary layer is significantly broadened, yielding incorrect pressure and temperature distributions, as seen in Figs. 2(a) and (b). The theoretical value 13.6405 of the wall temperature is obtained under the assumptions of adiabatic wall, $P_{r}=1.0$ and $O\left(R_{e}\right) \gg 1$. Several fixes for the Van Leer splitting have been proposed to resolve this issue of nonvanishing mass diffusion. Two types, in particular, have proved the most successful to date:

1. High Order Polynomial Expansions (HOPE) of the flux vector given by the authors [11], and a slight variation by Coirier and Van Leer [18], and

2. Hybrid FVS technique first proposed by Hänel et al [7-8] and later extended by Van Leer [9].

The former, while capable of generating a vanishing mass diffusion and producing excellent results for the conical 1D model problem, yields nonphysical oscillatory solutions in 2D problems. This is primarily due to the improper sign of the eigenvalues of the flux Jacobian in a small region in $-1 \leq M \leq 1$. The latter, while 
predicting correct boundary-layer thickness and wall temperature (see Fig. 3b), inflicts glitchs in the pressure near the edge of the boundary layer (see Fig. 3a), and incorrect sign of the normal velocity component near the wall, as will be seen later in Fig. 5. Clearly both improvements are still unacceptable.

The Roe splitting has been commonly accepted as one of the most accurate techniques available today. However the setup cost of this method requires $O\left(n^{2}\right)$ operations per grid point in each iteration, where $n$ is the number of equations. Thus a heavy price is paid for the accuracy obtained. Similar effort for reducing the computational cost has also been pursued in the Roe splitting. Roe [19] proposed to replace the dissipation matrix by the scalar amplitude-square weighted wave speed of Harten and Lax. Unfortunately, the scheme suffers from a stability problem in which the $L_{\infty}$ norm of the residual can be driven down only an order of magnitude on this test problem. One scheme essentially equal to the RS in accuracy is the Osher scheme. The major computation cost in this scheme lies in the determination of the intermediate states connecting the two states of the neighboring grid points. Again, $O\left(n^{2}\right)$ operations are required.

Based on the conclusions drawn from many past researches, one wonders whether it is possible to arrive at an upwind scheme with the following properties: (1) accurate; (2) simple, requiring only $O(n)$ operations; and (3) stable, for a wide range of problems. It is this question that motivates the research reported in the present paper.

\section{A New Upwind Scheme-AUSM:}

Now we present a new formulation of the numerical flux function which is neither a FVS nor a FDS technique. Simply put, the new scheme treats the convective and pressure terms separately. The convective terms are upstream-biased using a properly defined cell-interface velocity, while the pressure term is strictly dealt with by using acoustic waves. Accordingly, the name of the scheme is coined as Advec- 
tion Upstream Splitting Method (AUSM). The method retains the simplicity and efficiency of a FVS scheme, but achieves the high level of accuracy attributed only to FDS methods. Furthermore, it has proven thus far to be a robust formulation as a variety of Euler and Navier-Stokes calculations will bear out.

We turn now to the detailed derivation of the method. The first step is to recognize that the inviscid flux vector, $\mathbf{F}_{\text {inv }}$ consists of two physically distinct parts, namely convective and pressure terms:

$$
\mathbf{F}_{\text {inv }}=\left(\begin{array}{c}
\rho \\
\rho u \\
\rho v \\
\rho H
\end{array}\right) v+\left(\begin{array}{l}
0 \\
0 \\
p \\
0
\end{array}\right)=\mathbf{F}_{i n v}^{(c)}+\left(\begin{array}{l}
0 \\
0 \\
p \\
0
\end{array}\right) .
$$

The convective terms can be considered as passive scalar quantities convected by a suitably defined velocity $v$ at the cell interface. On the other hand, the pressure flux terms are governed by the acoustic wave speeds. Thus we propose to discretize the two components separately. The interface flux for supersonic flow is simple and set as usual by taking either the 'left' or 'right' state depending on the sign of the Mach number $M=v / a$. Thus, hereafter we will concentrate on the subsonic situation only, i.e., $-1 \leq M \leq 1$. At an interface $L<\frac{1}{2}<R$, the convective terms can be effectively written as:

$$
\mathbf{F}_{i n v_{1 / 2}}^{(c)}=v_{1 / 2}\left(\begin{array}{c}
\rho \\
\rho u \\
\rho v \\
\rho H
\end{array}\right)_{L / R}
$$

where

$$
(\bullet)_{L / R}= \begin{cases}(\bullet)_{L}, & \text { if } v_{1 / 2} \geq 0 \\ (\bullet)_{R}, & \text { otherwise }\end{cases}
$$

Notice that this development opens up a whole new family of schemes based upon the formulation chosen for the advective velocity $v_{1 / 2}$. One successful choice is to represent this velocity as a combination of the wave speeds $(v \pm a)$ traveling 
towards the interface (1/2) from the adjacent $L$ and $R$ cells. This is formally written as:

$$
v_{1 / 2}=a_{L / R} M_{1 / 2},
$$

where $M_{1 / 2}$ is defined by combining the contributions from both the 'left' and 'right' states, i.e.,

$$
M_{1 / 2}=M_{L}^{+}+M_{R}^{-} .
$$

Various ways of defining the split Mach numbers $M^{ \pm}$exist. In this paper we choose to use the Van Leer splitting [5]. For example, if $|M| \leq 1$, then

$$
M^{ \pm}= \pm \frac{1}{4}(M \pm 1)^{2} .
$$

Hence, the convective flux (3) becomes:

$$
\mathbf{F}_{i n v_{1 / 2}}^{(c)}=M_{1 / 2}\left(\begin{array}{c}
\rho a \\
\rho a u \\
\rho a v \\
\rho a H
\end{array}\right)_{L / R}
$$

All of the AUSM calculations performed for the four different experiments in this paper were completed using this formulation of the convective flux vector.

We turn now to the pressure term by writing:

$$
p_{1 / 2}=p_{L}^{+}+p_{R}^{-}
$$

Again considering the subsonic region, the pressure splitting is weighted using the polynomial expansion of the characteristic speeds $(M \pm 1)$. As observed in reference [10], the pressure splitting can be expressed in terms of second-order polynomials $(M \pm 1)^{2}$ as:

$$
p_{3}: \quad p^{ \pm}=\frac{p}{4}(M \pm 1)^{2}(2 \mp M) .
$$

The other expansion is the simplest possible form of the lowest order:

$$
p_{1}: \quad p^{ \pm}=\frac{p}{2}(1 \pm M) .
$$


In fact, a whole host of choices are possible for the pressure splitting. Having tested these two splittings thoroughly, we conclude that both perform admirably. However, upon close examination of the region downstream of a shock discontinuity, the third order pressure splitting $p_{3}$ can generate a small oscillation affecting the data by one cell. This will be discussed further in the next section. It is interesting to note that the above formulas can be recast in the following form:

$$
\left.\left(\begin{array}{c}
\rho v \\
\rho v u \\
\rho v v+p \\
\rho v H
\end{array}\right)_{1 / 2}=M_{1 / 2} \frac{1}{2}\left[\left(\begin{array}{c}
\rho a \\
\rho a u \\
\rho a v \\
\rho a H
\end{array}\right)_{L}+\left(\begin{array}{c}
\rho a \\
\rho a u \\
\rho a v \\
\rho a H
\end{array}\right)\right]_{R}\right]-\frac{1}{2}\left|M_{1 / 2}\right| \Delta_{1 / 2}\left(\begin{array}{c}
\rho a \\
\rho a u \\
\rho a v \\
\rho a H
\end{array}\right)+\left(\begin{array}{c}
0 \\
0 \\
p_{L}^{+}+p_{R}^{-} \\
0
\end{array}\right)
$$

where $\Delta_{1 / 2}\{\bullet\}=\{\bullet\}_{R}-\{\bullet\}_{L}$. Here the first term on the RHS is clearly not a simple average of ' $L$ ' and ' $R$ ' states, but rather a Mach-number-weighted average. Moreover, the dissipation term is merely a scalar $\left|M_{1 / 2}\right|$, requiring only $O(n)$ operations, in contrast to $O\left(n^{2}\right)$ operations by the Roe matrix.

Thus, the above splittings of both the advective term and pressure term completely define the Euler flux vector. For the viscous terms, the usual central-average representation is used at the interface.

\section{RESULTS AND DISCUSSION}

Four sets of problems were used to measure the performance of the new splitting technique. First there is the quasi 2-D viscous conical flow patterned after the experiments of Van Leer et al [6]. The second set of investigations involved the 2D inviscid calulation about a NACA 4-digit series airfoil. These tests were conducted under regimes similiar to the GAMM Workshop on the Numerical Simulation of Compressible Euler Flows [12]. The third problem was the shock wave and laminar boundary-layer interaction in which experimental measurements [13] were available for comparison. Finally, the fourth set dealt with a supersonic flow over a circular 
cylinder for which the Roe scheme is shown to yield anomalous solutions, depending on the Mach number and computation grid.

\section{Case 1:}

Let us consider a cone of 10-degree half angle with a computational domain spanning five degrees out from the surface of the cone. The freestream is defined by a Mach number of 7.95, Reynolds number of $4.2 \times 10^{5}$, stagnation temperature of $775.56 \mathrm{~K}$, and Prandtl number of 1.0. The case of unity Prandtl number was chosen so that the validation of the present scheme can be performed against the theoretical solution for the adiabatic wall temperature given by:

$$
\frac{T_{w a l l}}{T_{\infty}}=1+\frac{\gamma-1}{2} M_{\infty}^{2} .
$$

First we present first-order results by four techniques. Comparison of the results will clearly show the clue as to why some schemes fail. Next, second-order accurate calculations, using a two-step flux extrapolation procedure [14], were made to prove the stability of the present scheme and the further improvement in accuracy.

In Figs. 4 (a) and (b), the pressure and temperature distributions demonstrate the dramatic improvement by the AUSM from the VLS and related modifications in [8,9], which were shown earlier in Figs. 3 (a) and (b). It is gratifying to see that while extremely simple, the present AUSM results are essentially indistinguishable from that of the Roe splitting. Close analysis reveals, in Fig. 5, that the Van Leer scheme and subsequent modifications predict the normal velocity component near the wall with improper sign or value, in comparison with the Roe solution. The pressure irregularity is related to the strong change in the gradient of advective velocity component at the edge of the boundary layer. This suggests that consistent treatment ought be applied to all fluxes, especially the mass flux so that proper balance of velocity components is enforced. 
The second-order scheme, shown in Figs. 6 (a) and (b), further eliminates any remanent of the glitch in the pressure profile. As usual, the oscillation of the second-order result at the shock can be made to disappear by incorporating a standard TVD scheme. Note that the AUSM gives only very mild oscillations near the shock in the second-order result, weaker than the Roe solution.

In Figs. 7 (a) and (b), convergence history of the first- and second-order calculations for three splittings are shown. They all have more or less the same behavior and slope. However, it seems to indicate that the RS and AUSM are more similar. Also, the convergence rate does not appear deteriorated in the second-order calculations.

\section{Case 2:}

The NACA 0012 airfoil was chosen for inviscid calculations. The computational domain extends ten chord lengths out from the half-chord point, and is composed of $O$-type $97 \times 33$ cells. The code uses an explicit, six-stage Runge-Kutta integration scheme [15]. The finite volume formulation is second-order accurate, but does not include flux limiters. The experiment was conducted under two separate flow conditions: (a) $M_{\infty}=0.85$ and angle of attack $(\mathrm{AOA})=1.0$ degree; (b) $M_{\infty}=1.20$ and $\mathrm{AOA}=0.0$ degree.

Both cases demonstrate the AUSM as a robust flux splitting technique. In Figs. 8 (a), (b), and (c) we present the pressure contours and grid for case $2 a$ by the AUSM and RS. There is virtually no visible difference between the two solutions; both have accurately captured the upper and lower surface shocks. The solutions have converged at least four orders of magnitude.

The pressure coefficient at both surfaces for case $2 \mathrm{a}$ has also been plotted in Fig. 9 for both schemes; the lines are for the RS and the symbols for the AUSM. The AUSM appears to be slightly more accurate than Roe's by capturing the inviscid singularity, so called the Zierep singularity, at the foot of the shock 
on a curved surface. This is manifested by the overcompression followed by an expansion. However, both shocks are defined in the same number of cells.

The entropy plots for case 2a are displayed in Figs. 10 (a) and (b). It is interesting to note that the entropy generated at the leading edge is about three times weaker in magnitude and confined in a narrower region for the AUSM than the RS.

The pressure plots for case $2 \mathrm{~b}$ were presented merely to demonstrate the capability of the AUSM to capture the bow shock and the fish-tail shock; the accuracy is evident in Figs. 11 (a) and (b). Here, both results are essentially identical.

Up to this point, the cases 1 and 2 prove that the AUSM is as accurate as the RS. In the next two cases, we will begin to see significant differences, especially in the last case.

\section{Case 9:}

The experiment by Hakkinnen et al. [13] at $M_{\infty}=2.0, R e_{\infty}=2.96 \times 10^{5}$ and shock angle $=32.58^{\circ}$ was chosen for calculation using both the AUSM and RS. The computational domain consists of $75 \times 65$ grid points. While many calculations have been reported, there are substantial variations among these results as well as from the measurement. In the surface pressure and friction coefficient plots of Figs. 12 (a) and (b), we also see significant differences between two splittings. The AUSM gives very good agreement with the data [13] in $C_{f}$ in the separation as well as reattachment regions (solid dots are the separated region in which $C_{f}$ was not measured). For the surface pressure plotted in Fig. 12(a), the AUSM again produces good agreement with the measurement in the reattachment region, but under-predicts in the separation region. This point is particularly puzzling since the pressure rise and the drop in skin friction should go hand in hand. There is no apparant reason that a prediction coincides with the $C_{f}$, but not with $P$. However, the ability of obtaining good agreement of the reattachment with the data is worth 
noting since it is usually harder to achieve this than to predict separation. Further investigation of the experiment is clearly useful, but this is beyond the scope of the present paper. Insofar as the uncertainty in the measurements, it is hard to draw definitive judgment regarding the accuracy of both schemes. Figures 13 (a) and (b) display the Mach contours; the AUSM results seem to give a sharper definition of the incoming oblique shock as well as the complex expansion/compression waves in the interaction region. We note that residuals have dropped five orders of magnitude in both caculations.

\section{Case 4:}

The 2D supersonic flow over a circular blunt body was chosen to demonstrate the ability of the AUSM to correctly resolve the strong bow shock and the acceleration of stagnant flow through sonic point to supersonic speed. The same code used for case 2 was used here, but only with the first-order option to test the scheme's minimum capability for this type of problem. A range of free-stream Mach numbers from 2 to 20 were calculated along with the study of grid effect on the solution. The results presented were obtained from two types of grids: (1) $57 \times 33$ circular grid with stretching in the radial direction, and (2) $45 \times 43$ shock-aligned grid with uniform radial spacing; they are displayed together with the Mach number contours for the calculations to be presented.

As reported recently by Peery and Imlay [16] and Lin [17], Roe's splitting produced anomalous solutions that were manifested by odd-shaped bow shocks. This nonphysical solution, sometimes referred to as the "carbuncle phenomenon", is not completely understood yet. In our calculations, three types of "carbuncle phenomena" have been observed in the contours. They are nonsymmetric, protuberant, and indented. The solutions appeared to be grid and Mach number dependent. Several different Mach numbers were examined on several different grids. In the following, we show some typical results obtained by the Roe splitting and AUSM. 
Figures 14 (a) and (b) give the comparison of Mach contours for $M_{\infty}=6.0$ along with the grid in Fig. 14(c). For the RS, a protuberant shock is stopped at the outer boundary and a nonsymmetric flow is developed near the stagnation point. A velocity vector plot in this enlarged region, Fig. 13(d) shows a complex but nonphysical flow pattern. The AUSM on the other hand results in a smooth shock profile. It is noted that all results reported for this case have been obtained with residual dropped more than five decades for both schemes.

Figures 15 (a) and (b) display the results for $M_{\infty}=4.0$. The RS yields a nonsymmetric flow while the AUSM converges to a completely symmetric solution. However, a slight irregularity seen on the sonic line was attributed to the use of the pressure splitting, $p_{3}$ in (8a). Close investigation of the staircase contours in the curved part of the shock revealed that they are largely due to the nonalignment of the shock with the circular grid (Fig. 15(c)). This effect disappears as the grid is aligned with the shock, as seen in the previous test, Figs. 14.

A change of the Mach number to $M_{\infty}=3.0$ on the same grid resulted in a different type of contour in the RS calculation. Indented but symmetric contours, Fig. 16(b), were developed. This shows the sensitivity of the RS solution to the flow condition. Again, the AUSM gives correct behavior, except a slight glitch on the sonic line due to the $p_{3}$ splitting.

Next, we show the effect of the grid on the solution while the Mach number $M_{\infty}=6.0$ is kept the same as that in Figs. 14. In Figs. 17, the RS solution changed to the indented type from the opposite, protuberant type. Once again, the AUSM behaved rather remarkably.

Finally, we show the effect of pressure splittings, (8a) and (8b), on the AUSM solution. Shown in Fig. 18 is the pressure distribution along the cells immediately next to the symmetry line, with which the cell interface coincides. One post-shock overshoot is seen. It must be stressed that this overshoot is different from the 
oscillations seen normally either from the central differencing or higher-order upwind differencing, for there is no noise ahead of the shock. This shows that in this problem the AUSM captures the normal shock in two cells. Thus, inaccuracy (smearing) of the shock profile lies in these two cells and whether they are under- or over-shoot should be regarded as equally inaccurate. The $p_{1}$ splitting is seen to give a much milder overshoot and a different shock location by one cell. Furthermore, the shock location is more accurately predicted by $p_{1}$, as compared with the result from the grid refinement study.

\section{CONCLUDING REMARKS}

As guided by physical intuition and mathematical property, we propose a new flux splitting formula, AUSM, that uses a properly defined advection velocity to determine an upstream extrapolation at the cell interface. The scheme is remarkably simple, requiring only $O(n)$ operation, and renders itself for an easy implementation in a code. Furthermore, it is at least as accurate as the Roe solver for the problems tested. In the blunt body problem, the AUSM resulted in correct solutions without difficulty in every test in a wide variation of flow conditions and grids where the Roe splitting failed. The above promising features should make the new scheme very suitable for a calculation requiring both efficiency and accuracy, e.g., in the case of Navier-Stokes or chemical reacting flows. Our search for an alternative splitting that will meet the goals of efficiency, accuracy, and robustness does suggest that there are sufficient possibilities for further success.

\section{REFERENCES}

1. M.-S. Liou, and B. van Leer, AIAA paper 88-0624, 1988 (unpublished).

2. B. Koren, J. Comput. Phys. 87, 25 (1990).

3. A. Suresh and M.-S. Liou, to appear in AIAA J. (1991). 
4. A. Suresh and M.-S. Liou, "The Osher Scheme for Non-Equilibrium Reacting Flows," (submitted to Int. J. Numer. Methods Fluids).

5. B. van Leer, Lecture Notes in Physics 170 (Springer-Verlag, New York/Berlin 1982), p.507.

6. B. $\operatorname{van}$ Leer, J. L. Thomas, P. L. Roe, and R. W. Newsome, AIAA paper 87-1104, 1987 (unpublished).

7. D. Hänel, R. Schwane, and G. Seider, AIAA paper 87-1105, 1987 (unpublished).

8. D. Hänel and R. Schwane, AIAA Paper 89-0274, 1989 (unpublished).

9. B. van Leer, NASA CP-3078, p.203, 1991 (unpublished).

10. M.-S. Liou, B. van Leer, and J.-S. Shuen, J. Comput. Phys. 87, 1 (1990).

11. M.-S. Liou and C. J. Steffen, Jr., To appear in Proceedings, International Conference on Computational Engineering Science 1991, Melbourne, Australia.

12. "GAMM Workshop on the Numerical Simulation of Compressible Euler Flows," June 1986.

13. R. J. Hakkinen, I. Greber, L. Trilling, and S. S. Abarbanel, NASA Memo 218-59W, 1959 (unpublished).

14. C. Hirsch, Numerical Computation of Internal and External Flows, Vol. 2 (Wiley and Sons, 1990), p.495.

15. B. van Leer, C.-H. Tai, and K. G. Powell, AIAA paper 89-1933, 1989 (unpublished).

16. K. M. Perry and S. T. Imlay, AIAA paper 88-2904, 1988 (unpublished).

17. H.-C. Lin, AIAA paper 91-1544, 1991 (unpublished).

18. W. J. Coirier and B. van Leer, AIAA paper 91-1566, 1991 (unpublished).

19. P. L. Roe, ICASE Report 84-53, 1984 (unpublished). 


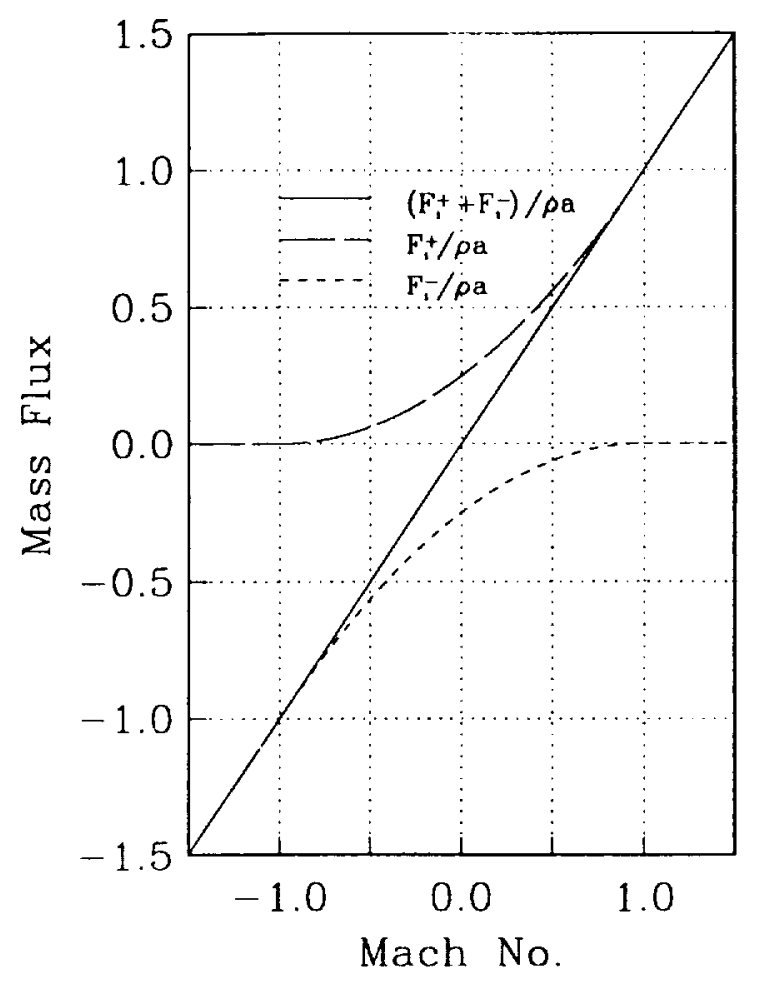

Fig. 1. Mass flux splitting of the Van Leer scheme.
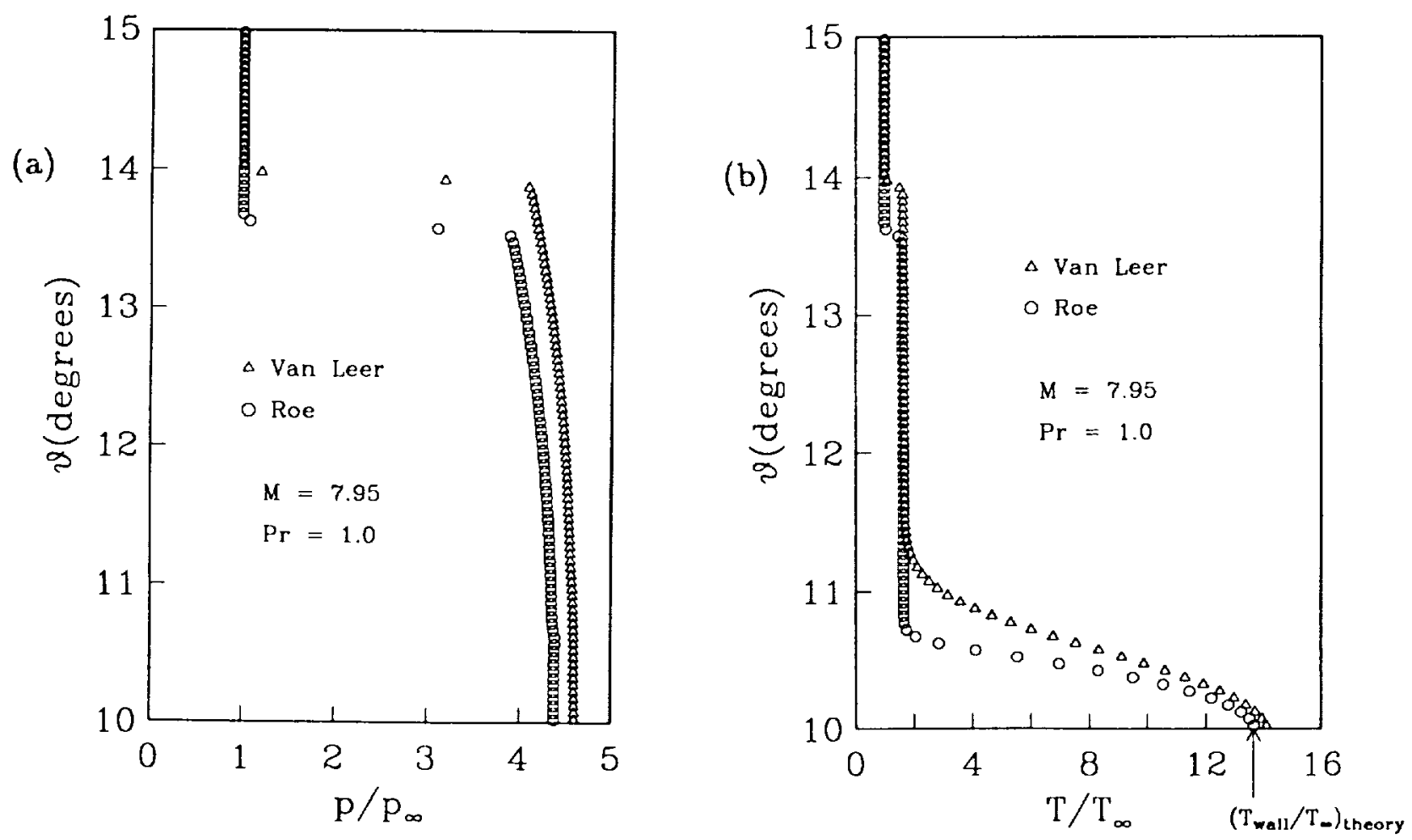

Fig. 2. Hypersonic conical flow, first order accurate results: (a) pressure profile and (b) temperature profile, using the Van Leer and Roe splittings. Note the diffused boundary layer of the Van Leer solution and its effect on shock location. 

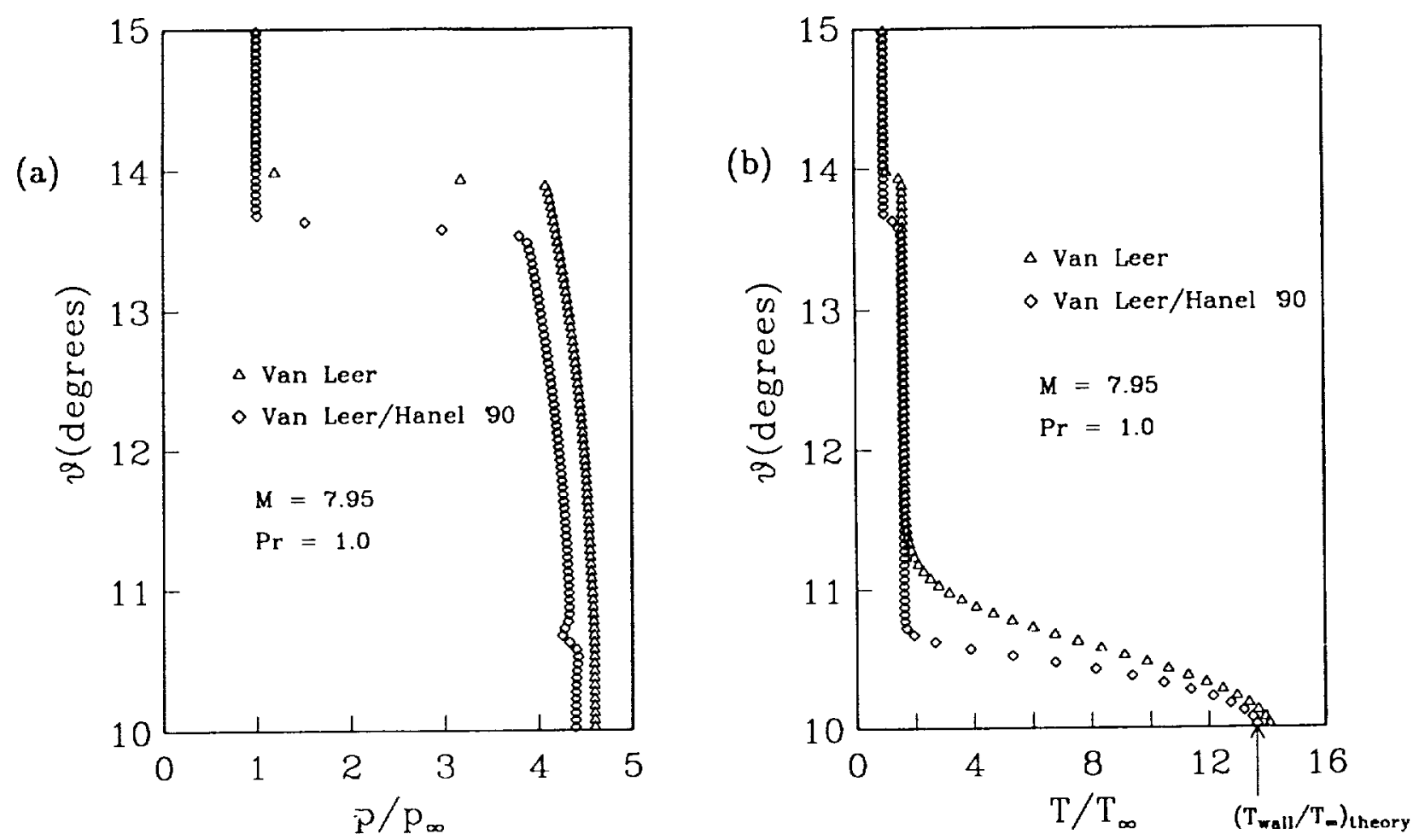

Fig. 3. Hypersonic conical flow, first order accurate results: (a) pressure profile and (b) temperature profile, using the Van Leer and the modified (Hänel/Van Leer) splittings.
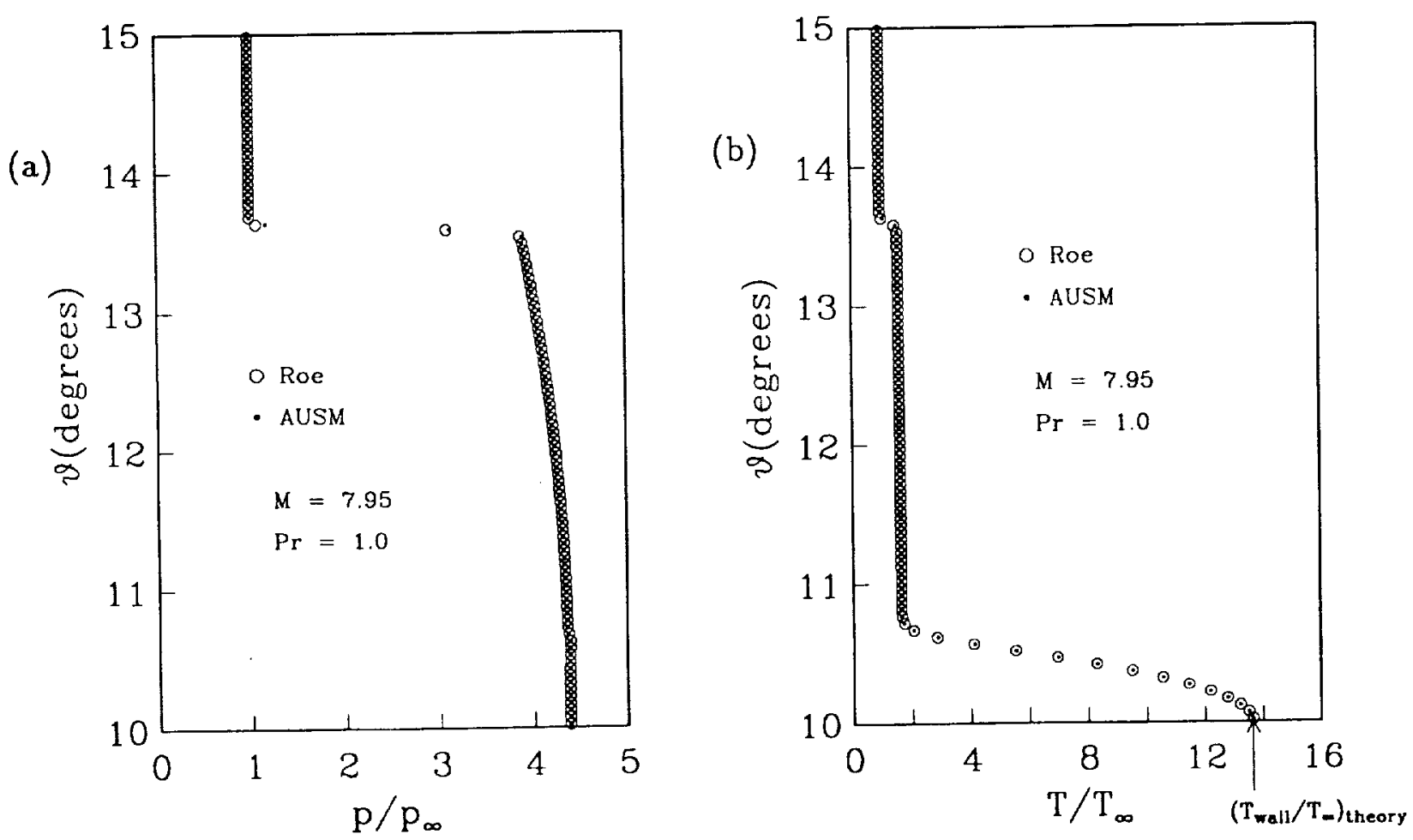

Fig. 4. Hypersonic conical flow, first order accurate results: (a) pressure profile and (b) temperature profile, using the Roe and present splittings. 


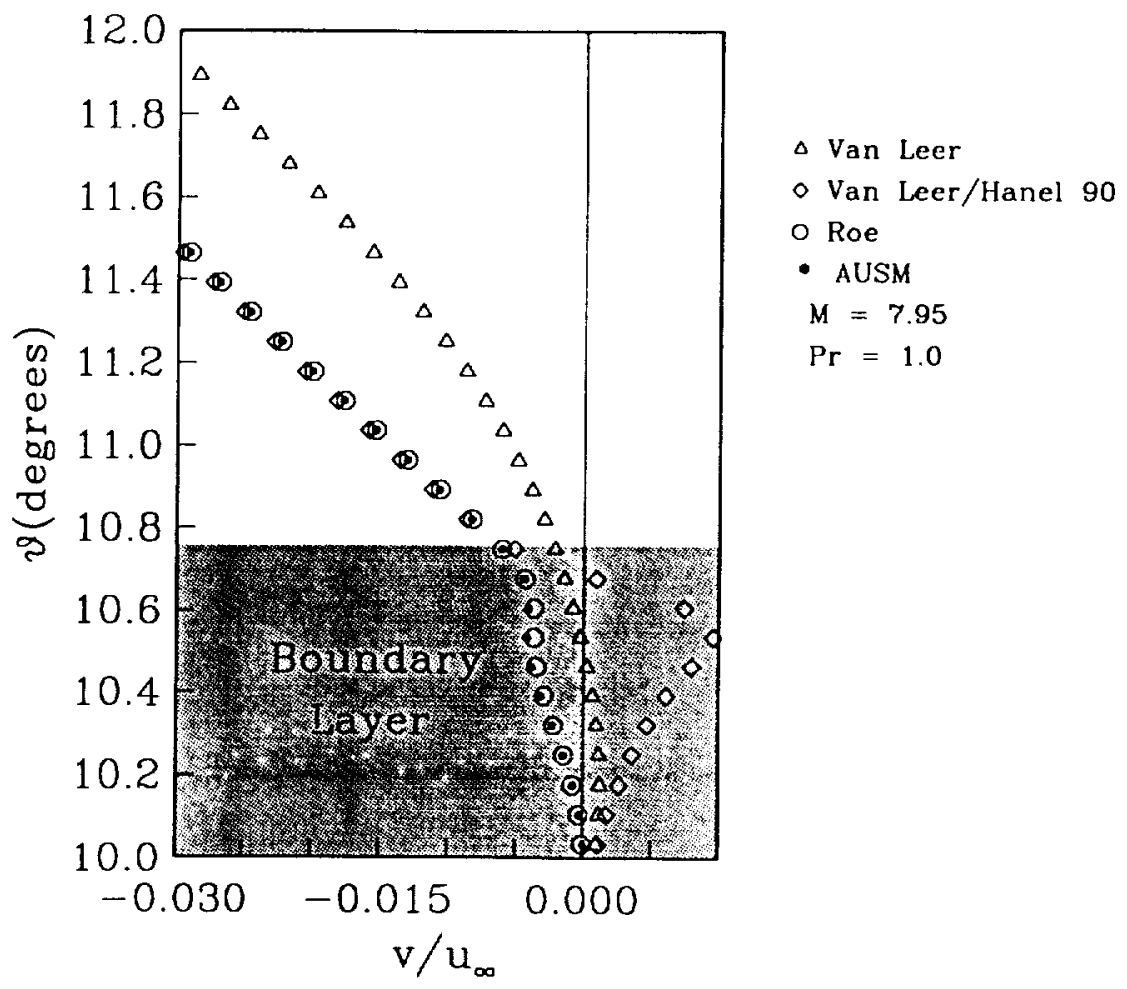

Fig. 5. Hypersonic conical flow, first order accurate results: the transverse velocity profiles of all four splittings.
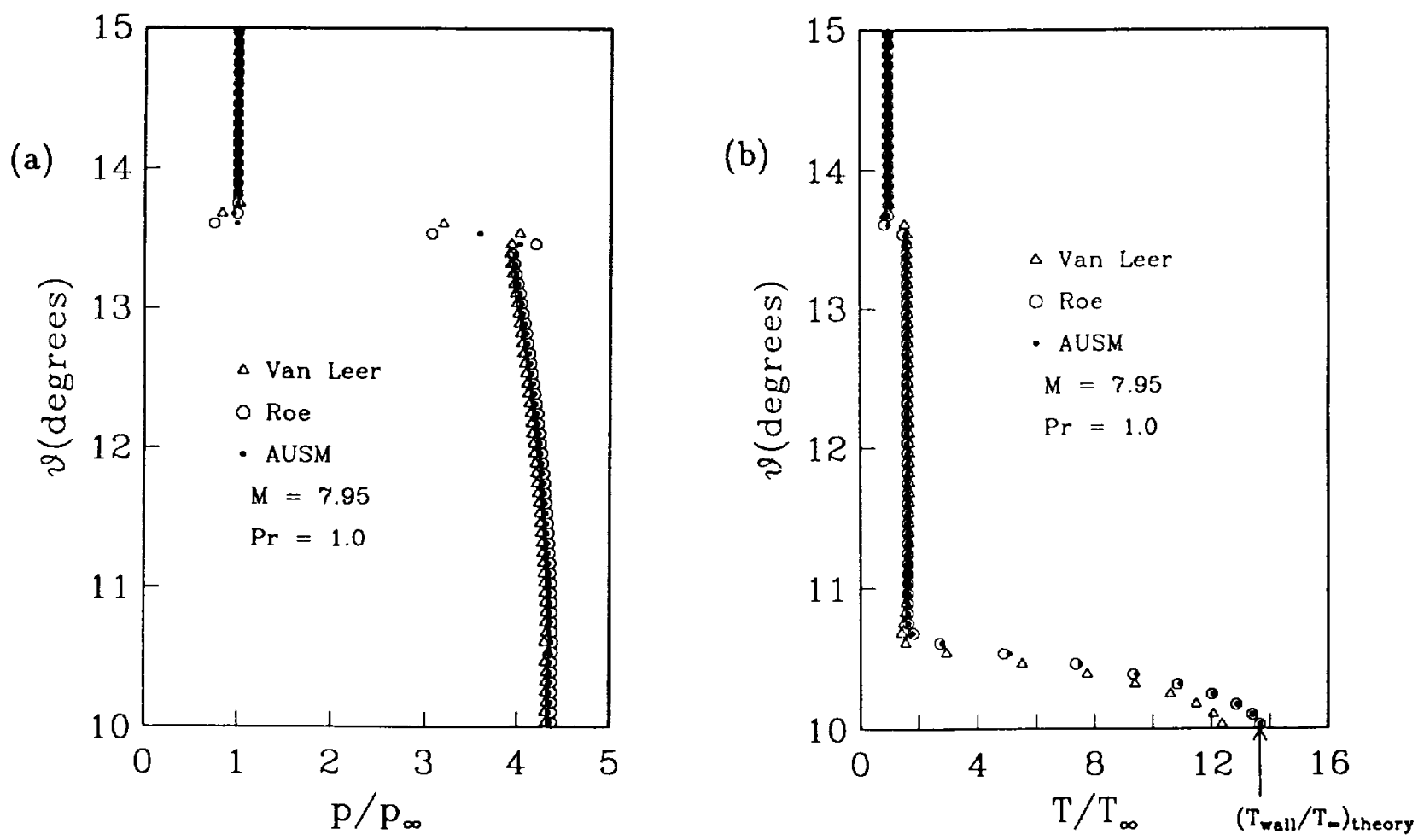

Fig. 6. Hypersonic conical flow, second order accurate results: (a) pressure profile and (b) temperature profile, using the Van Leer FVS, Roe FDS, and AUSM. 

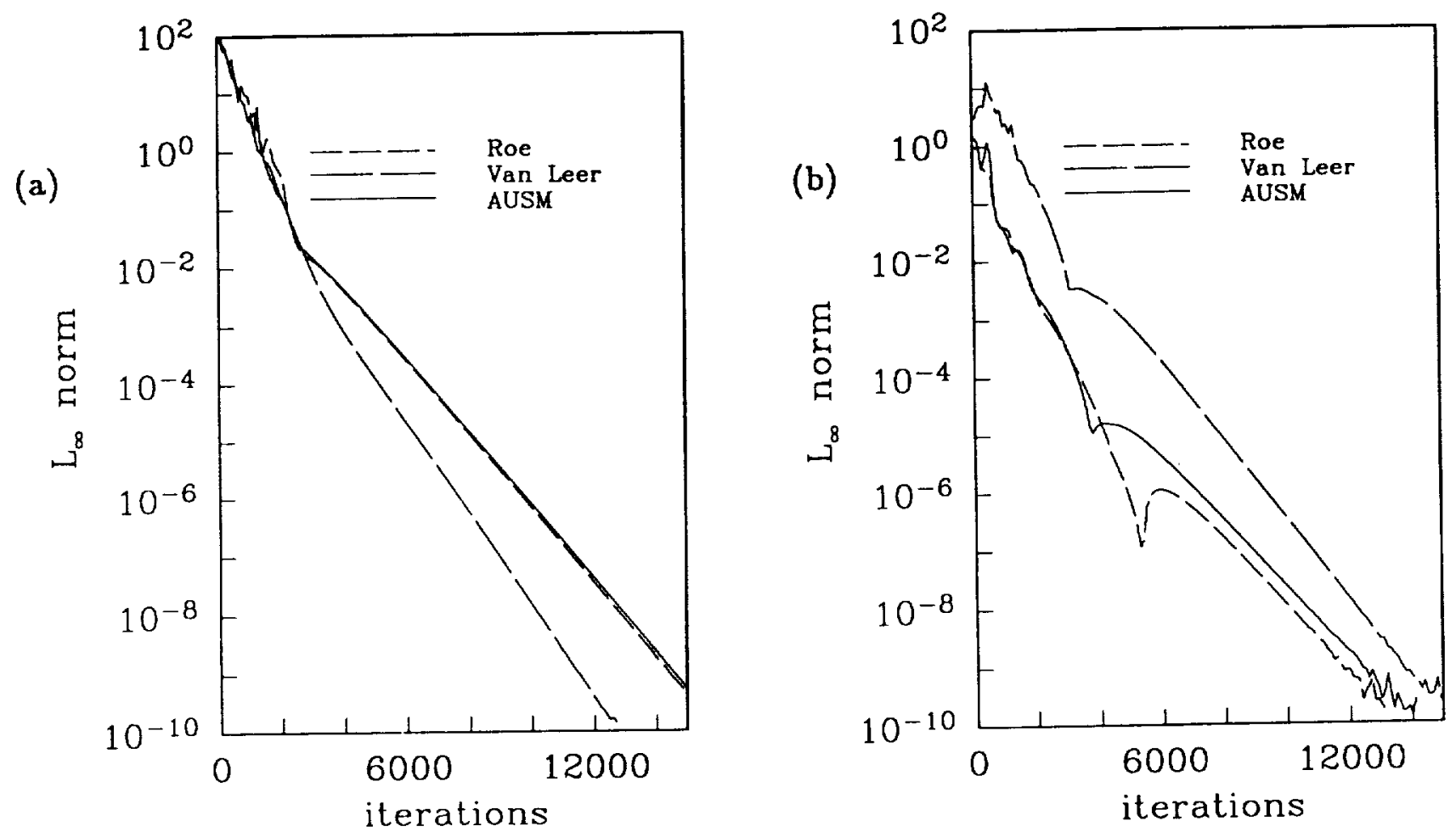

Fig. 7. Hypersonic conical flow, convergence histories: (a) first order accurate schemes and (b) second order accurate schemes. 


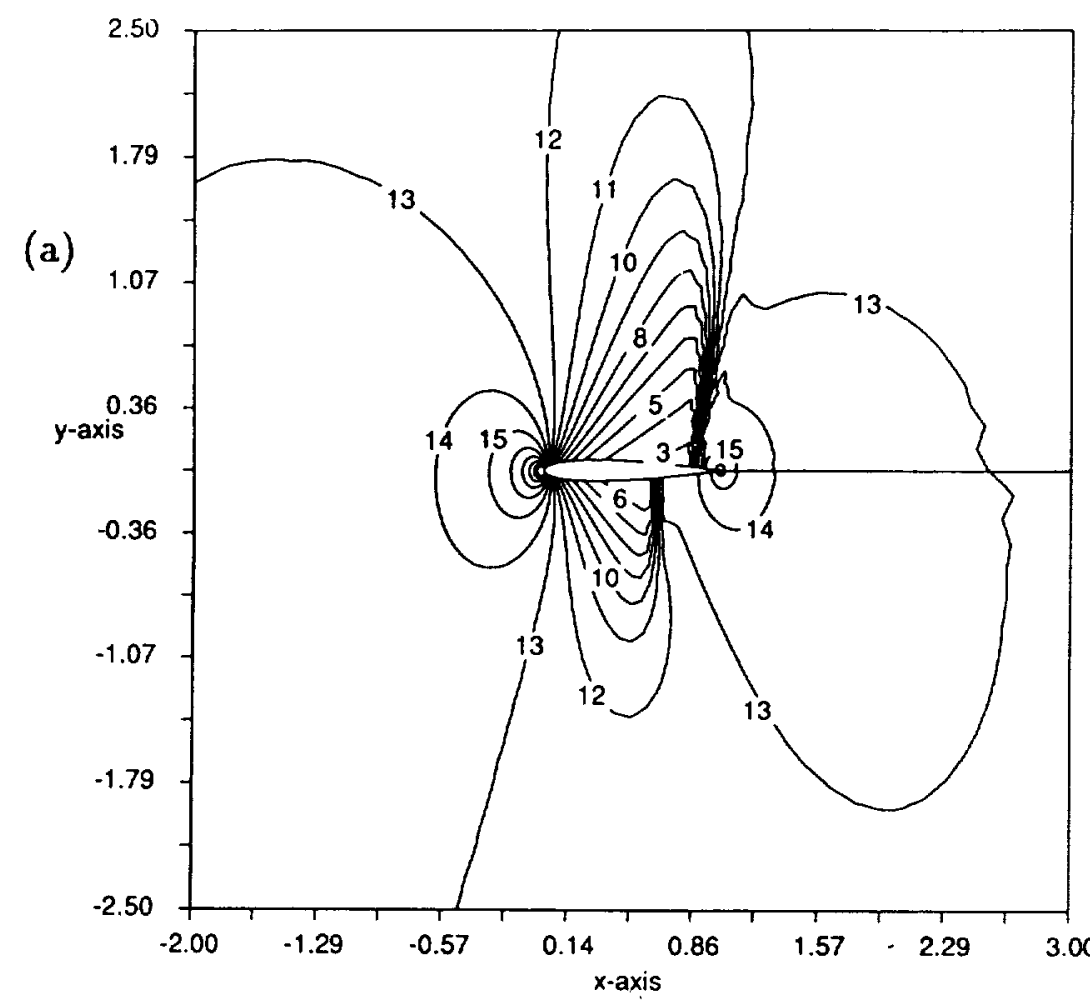

(c)

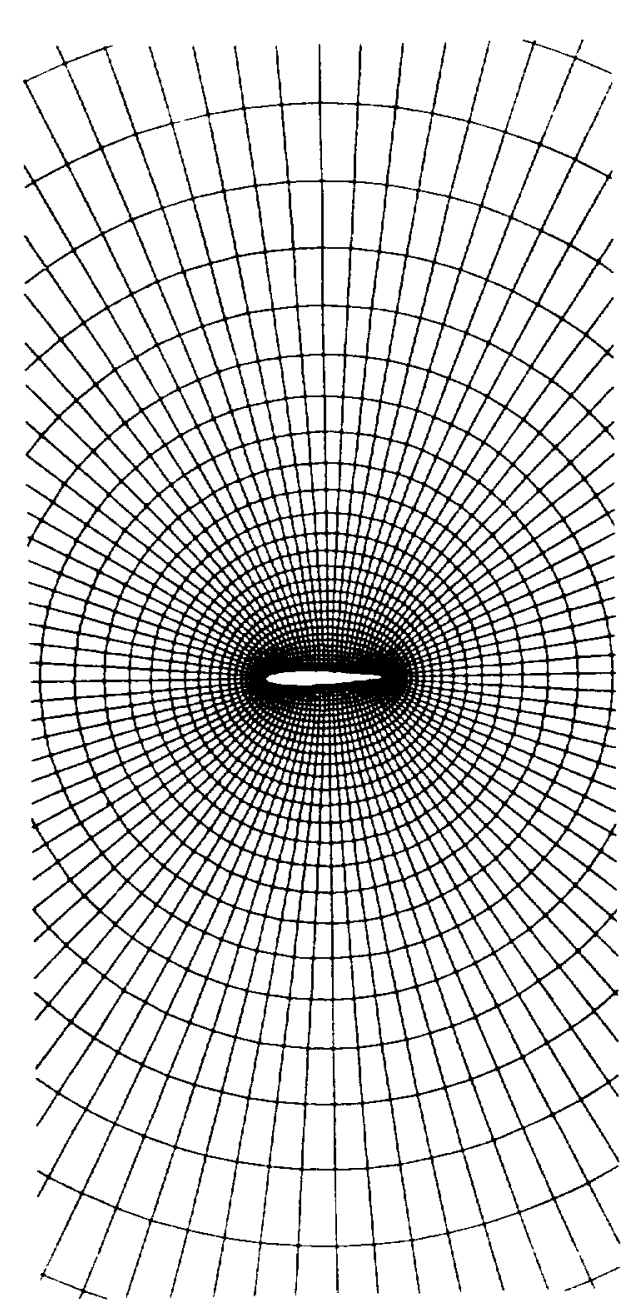

(b)

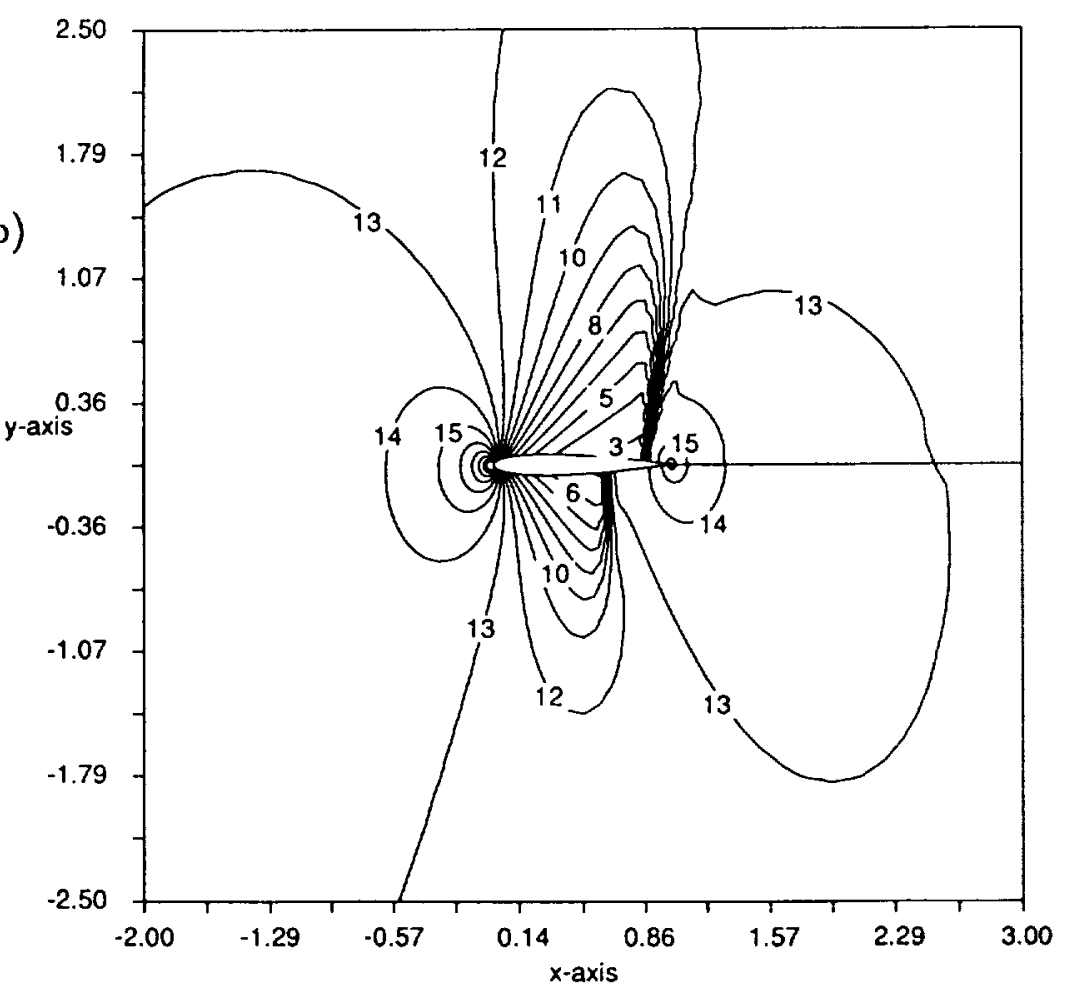

10.4000

20.4500

$3 \quad 0.5000$

40.5500

50.6000

60.6500

0.7000

$8 \quad 0.7500$

90.8000

$10 \quad 0.8500$

110.9000

Fig. 8. NACA 0012 transonic flow, pressure contours: (a) AUSM and (b) Roe FDS. The $97 \times 33$ grid is shown in (c). 


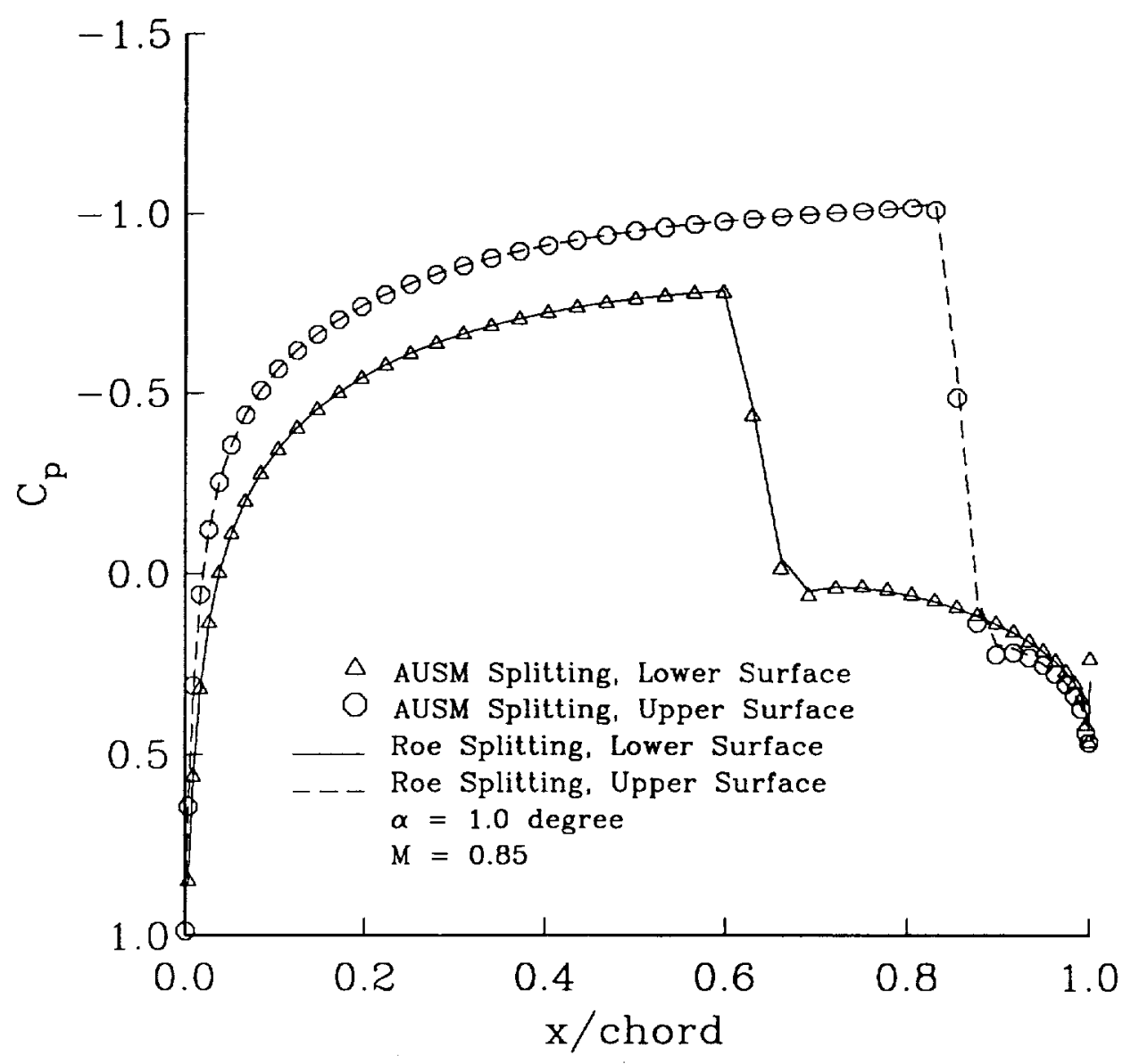

Fig. 9. NACA 0012 transonic flow, surface pressure coefficient. 
(a)

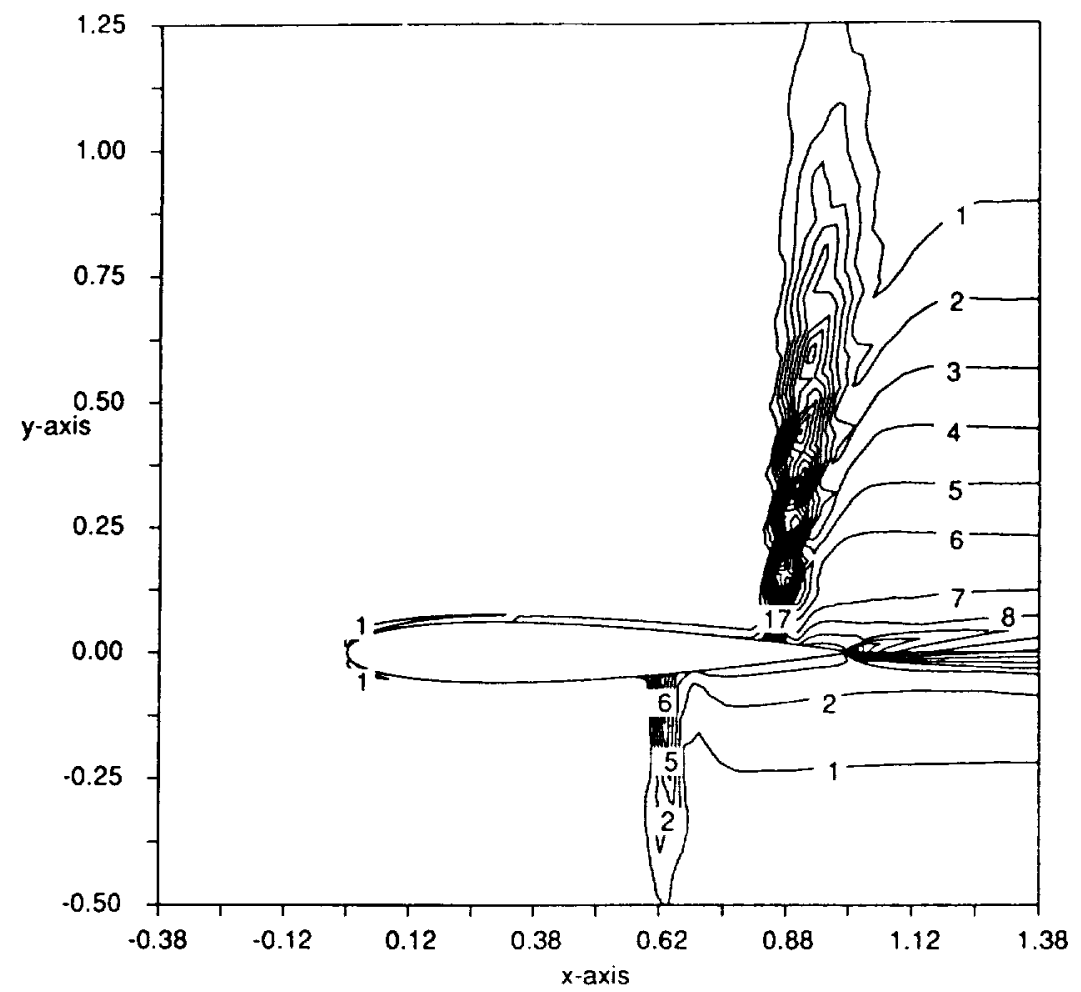

(b)

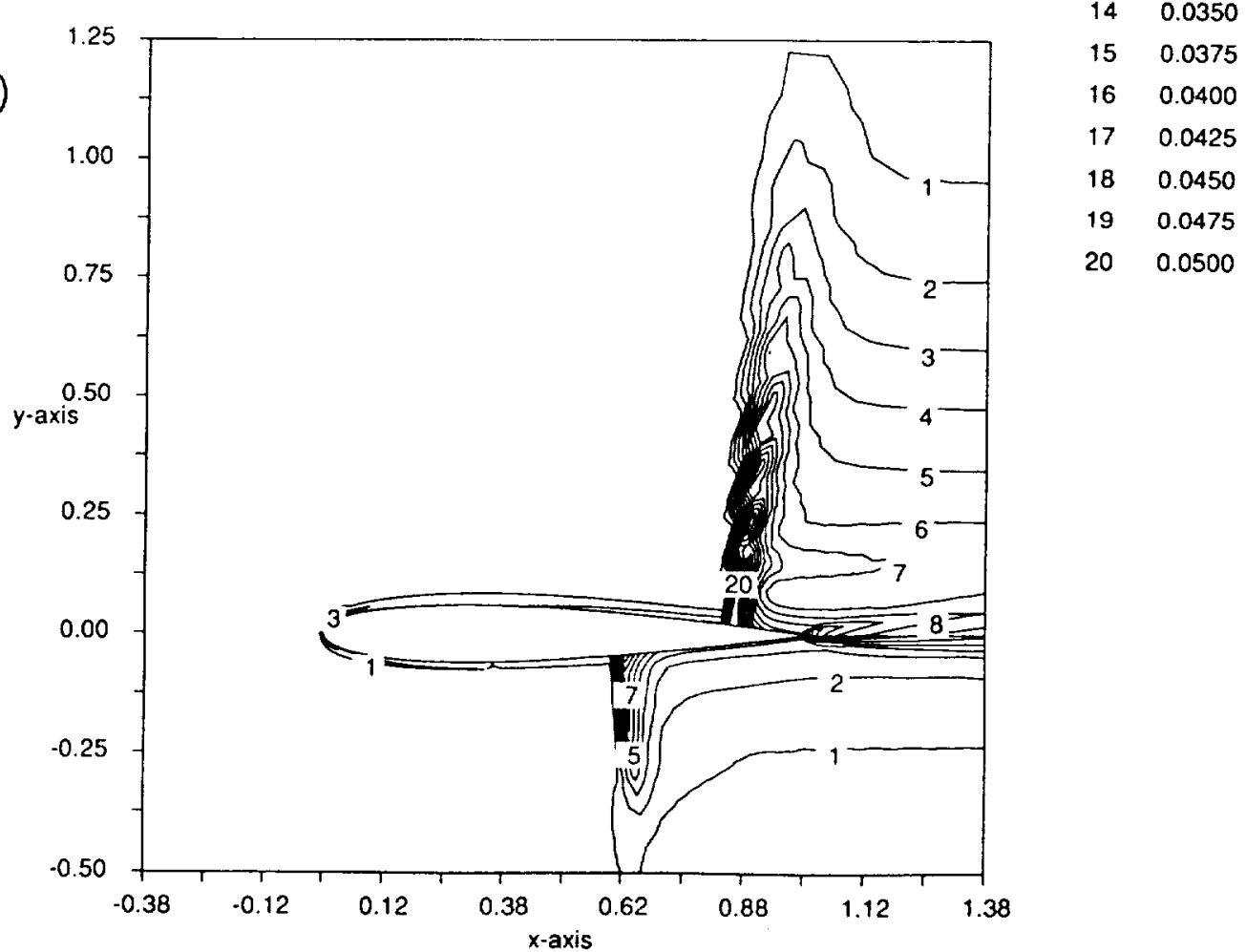

Fig. 10. NACA 0012 transonic flow, entropy contours: (a) AUSM and (b) Roe FDS. 


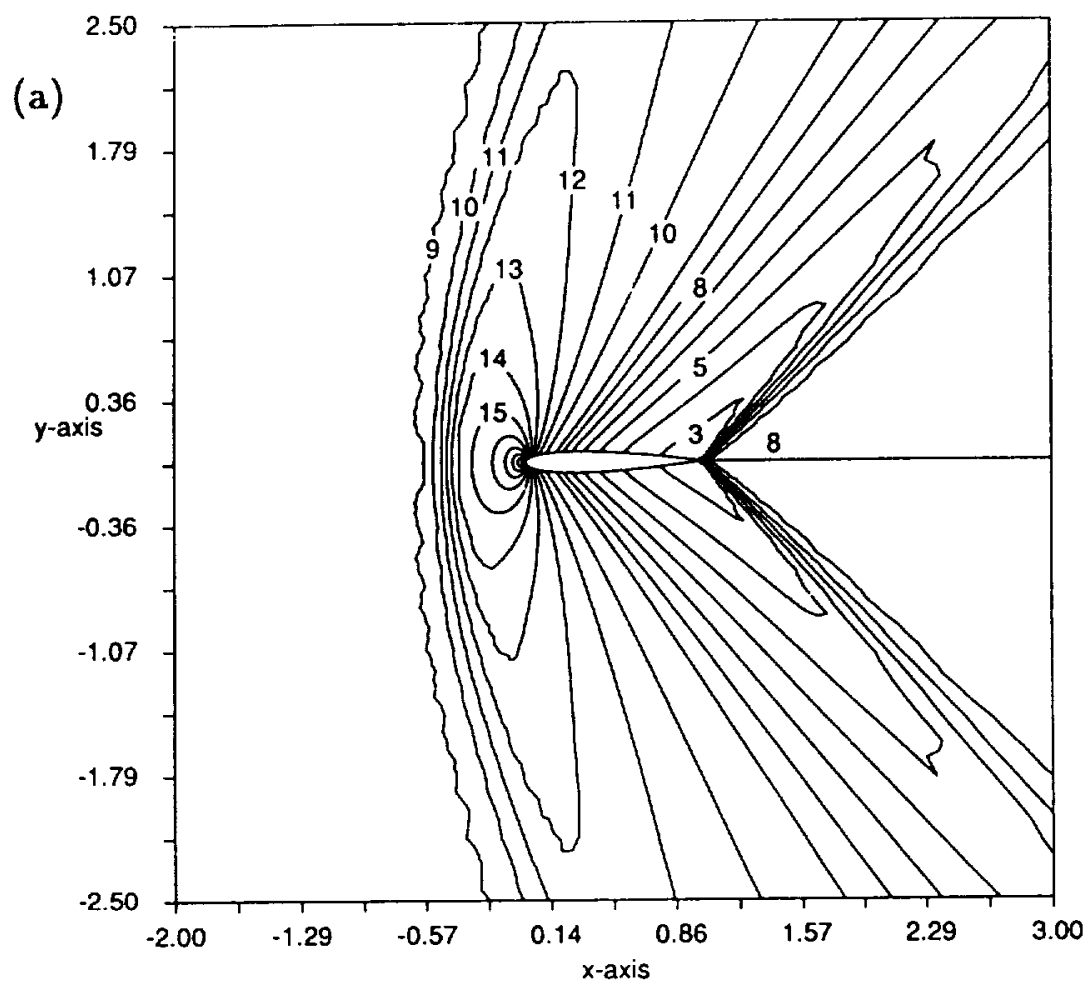

$\begin{array}{cc}1 & 0.3000 \\ 2 & 0.3250 \\ 3 & 0.3500 \\ 4 & 0.3750 \\ 5 & 0.4000 \\ 6 & 0.4250 \\ 7 & 0.4500 \\ 8 & 0.4750 \\ 9 & 0.5000 \\ 10 & 0.5500 \\ 11 & 0.6000 \\ 12 & 0.6500 \\ 13 & 0.7000 \\ 14 & 0.7500 \\ 15 & 0.8000 \\ 16 & 0.8500 \\ 17 & 0.9000 \\ 18 & 0.9500 \\ 19 & 1.0000 \\ 20 & 1.0500\end{array}$

(c)
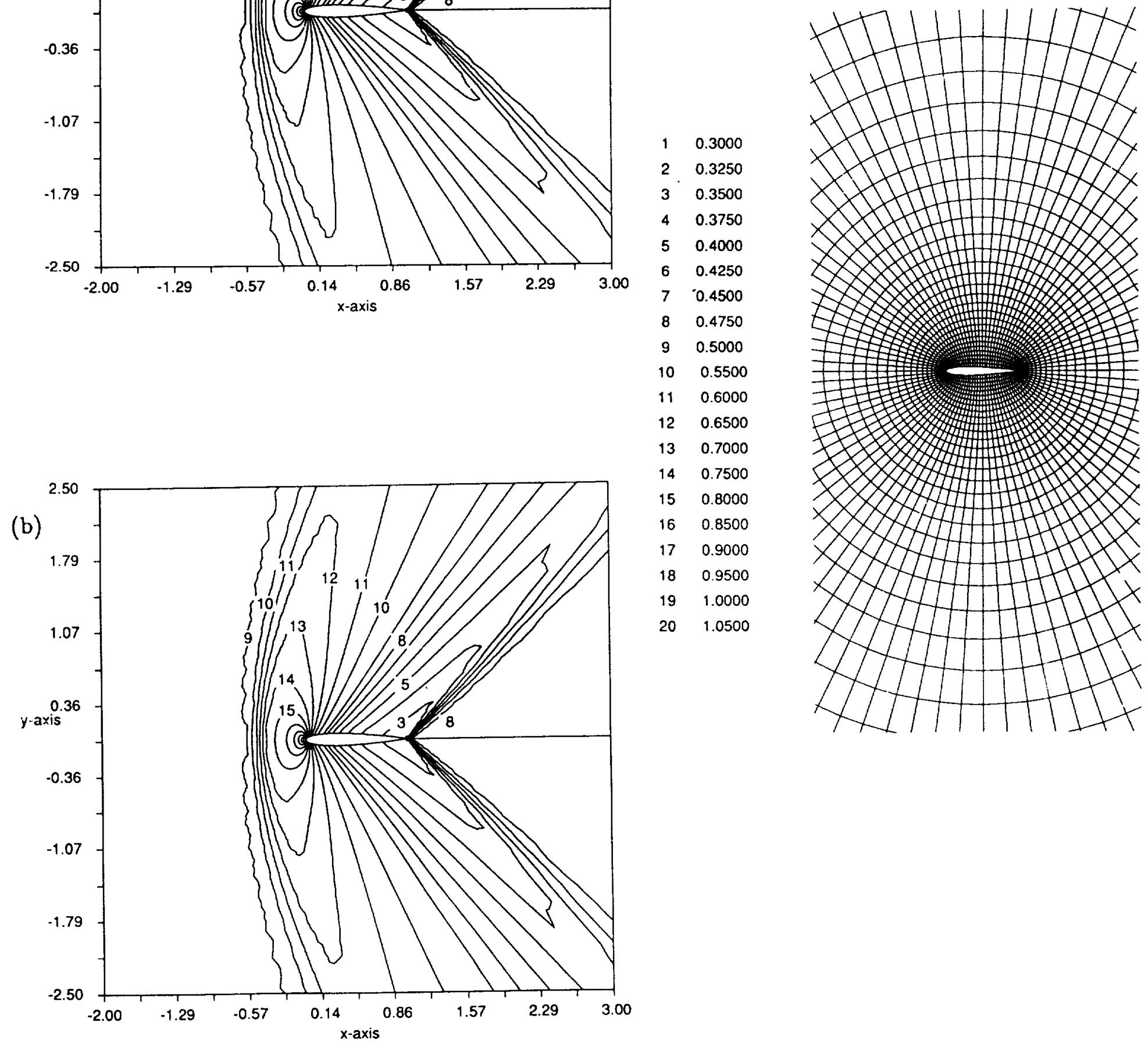

Fig. 11. NACA 0012 supersonic flow, pressure contours: (a) AUSM and (b) Roe FDS. The $97 \times 33$ grid is shown in (c). 

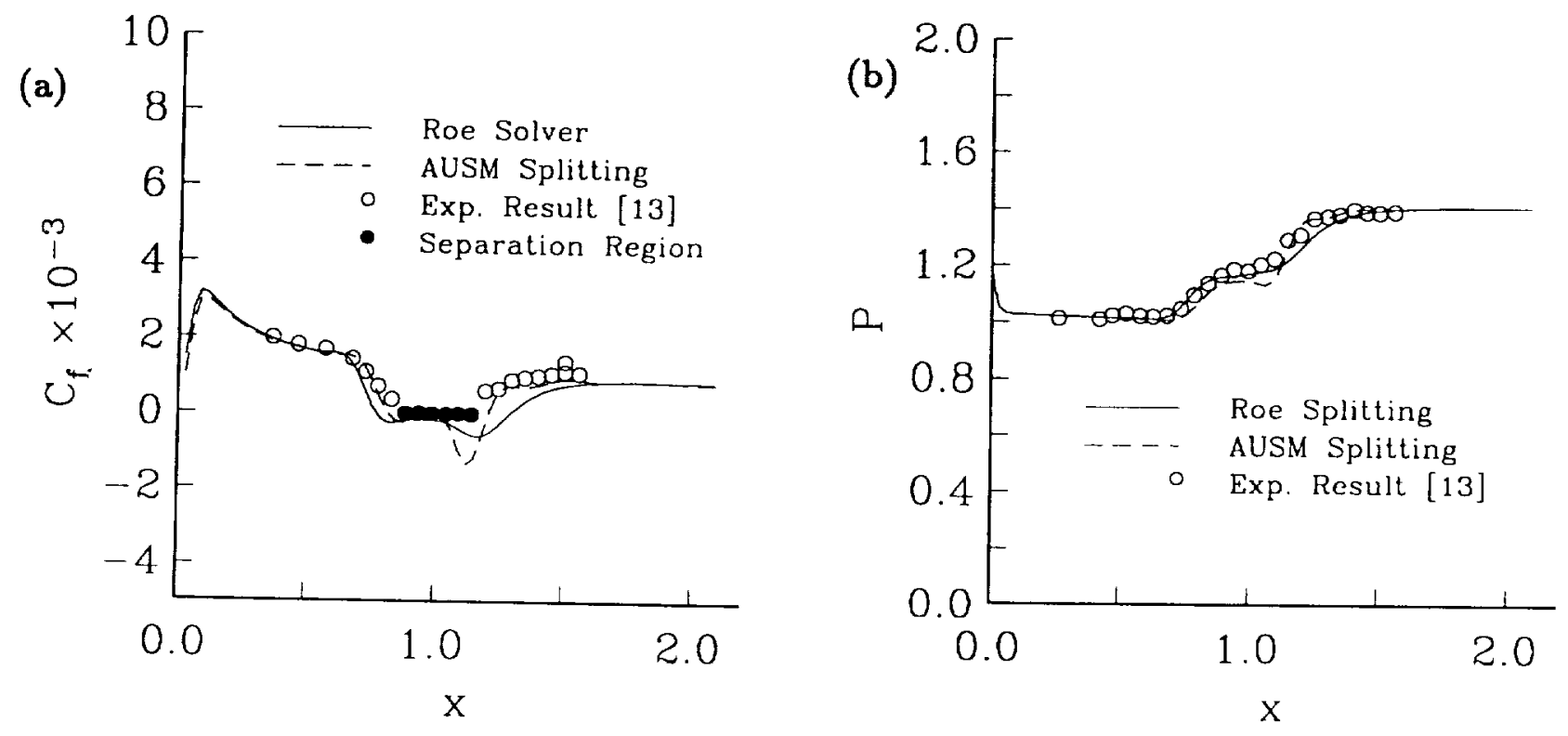

Fig. 12. Shock wave and boundary layer interaction: (a) skin friction coefficient and (b) pressure at the wall of the Roe FDS and AUSM solutions in comparison to experimental data [13]. 
(a)

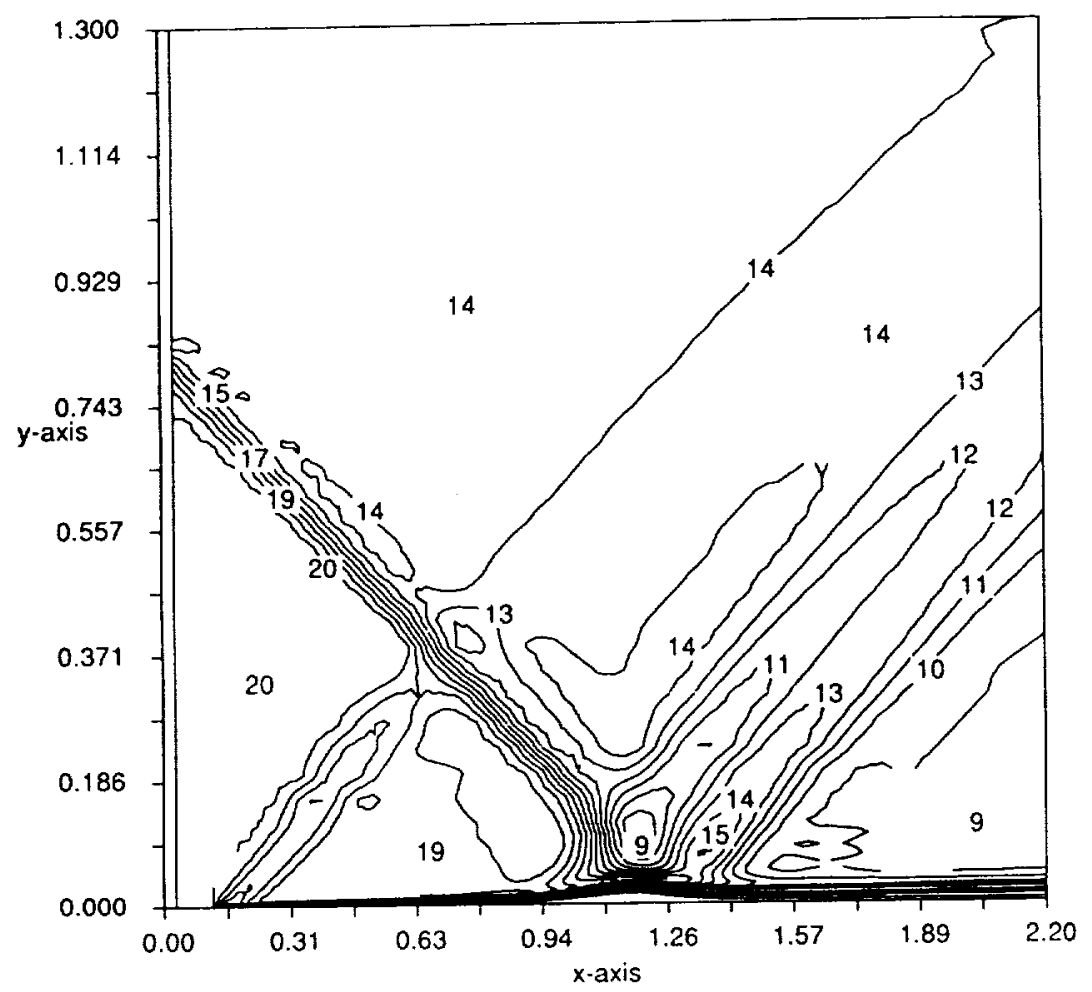

(b)

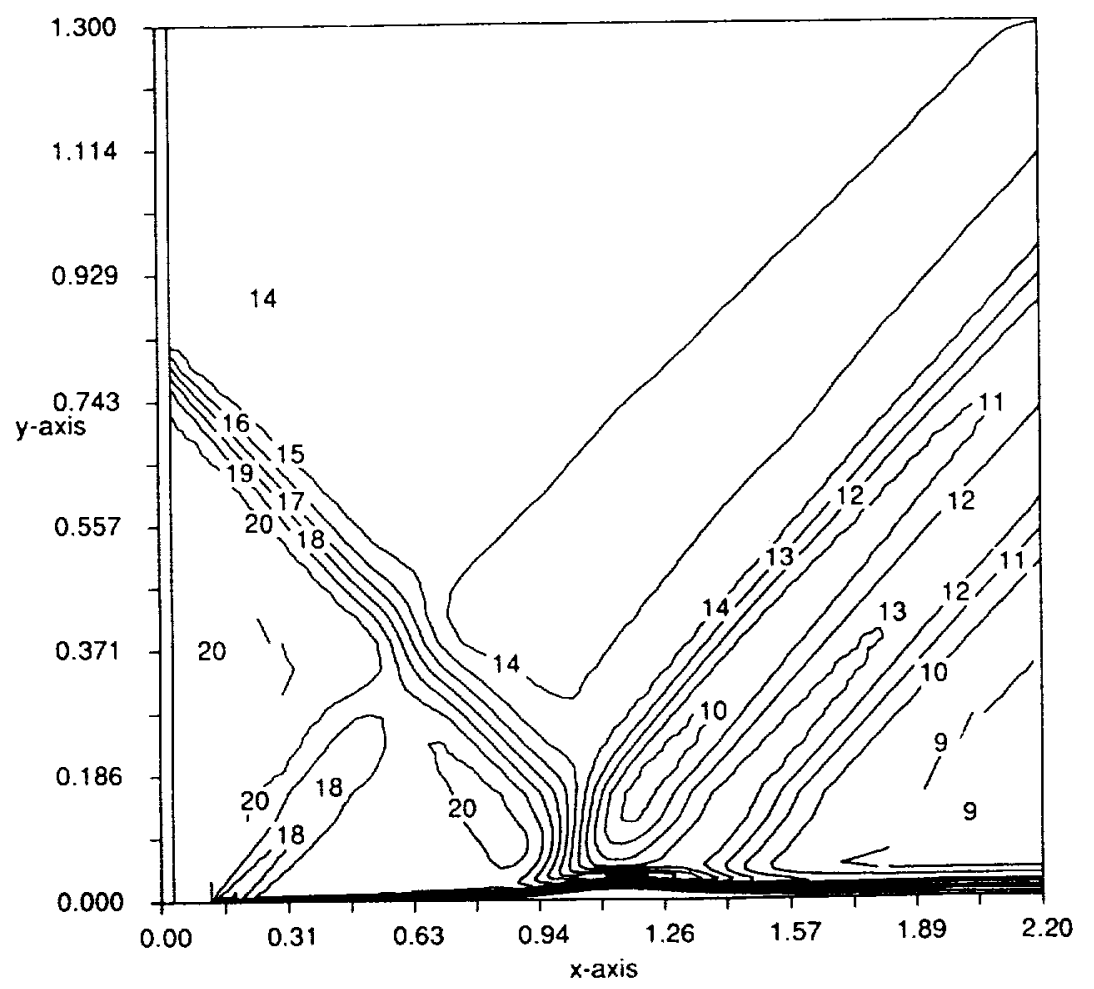

\begin{tabular}{c|c}
1 & 0.5000 \\
2 & 1.0000 \\
3 & 1.2500 \\
4 & 1.5000 \\
5 & 1.7000 \\
6 & 1.7200 \\
7 & 1.7400 \\
8 & 1.7600 \\
9 & 1.7800 \\
10 & 1.8000 \\
11 & 1.8200 \\
12 & 1.8400 \\
13 & 1.8600 \\
14 & 1.8800 \\
15 & 1.9000 \\
16 & 1.9200 \\
17 & 1.9400 \\
18 & 1.9600 \\
19 & 1.9800 \\
20 & 2.0000
\end{tabular}

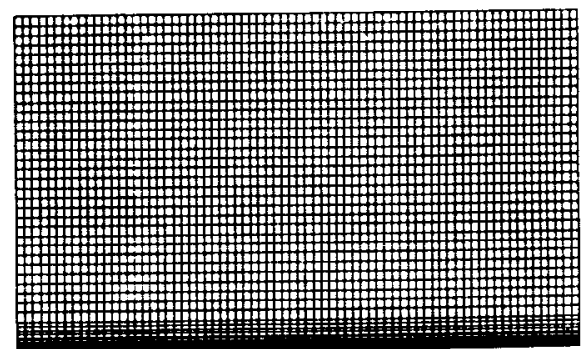

Fig. 13. Shock wave and boundary layer interaction, Mach number contours: (a) AUSM and (b) Roe FDS. The $75 \times 65$ grid is shown in (c). 
(a)

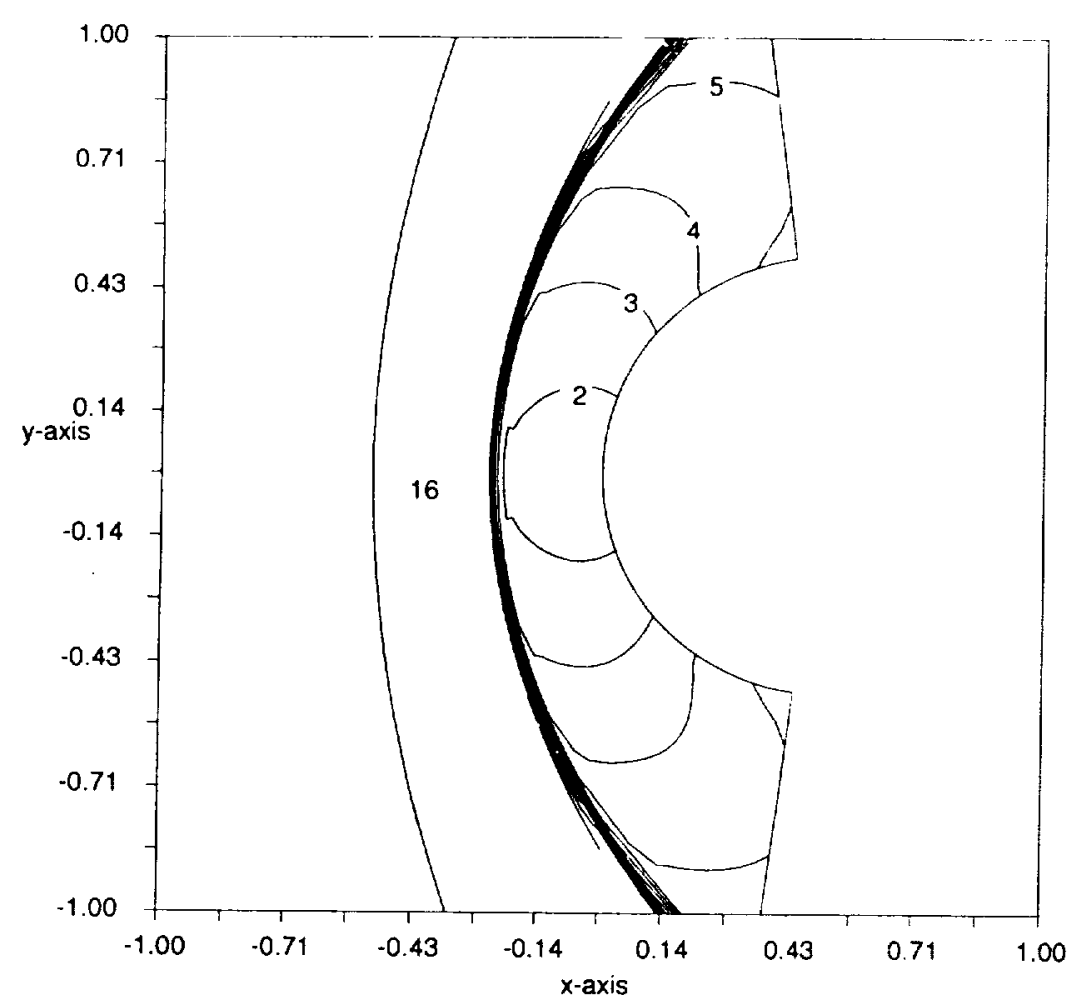

(c)
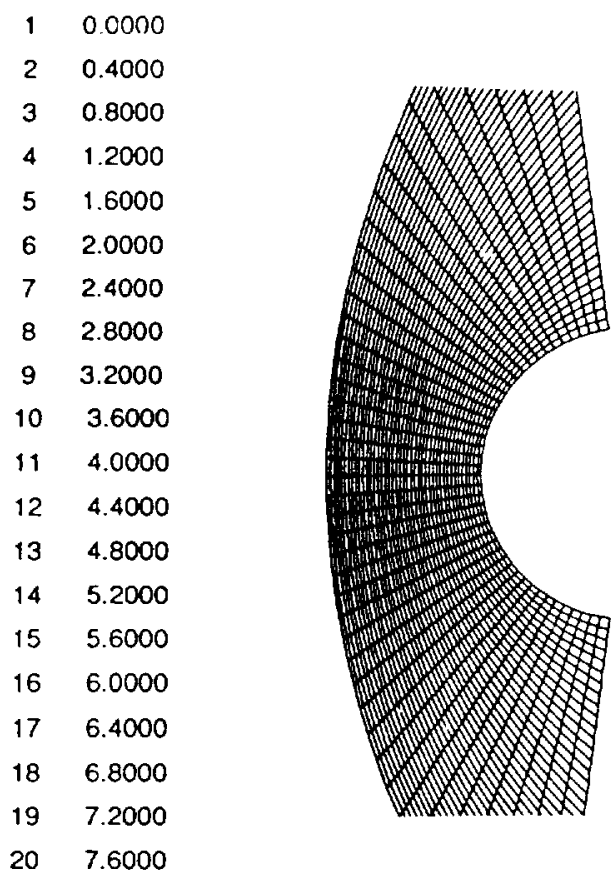

(b)

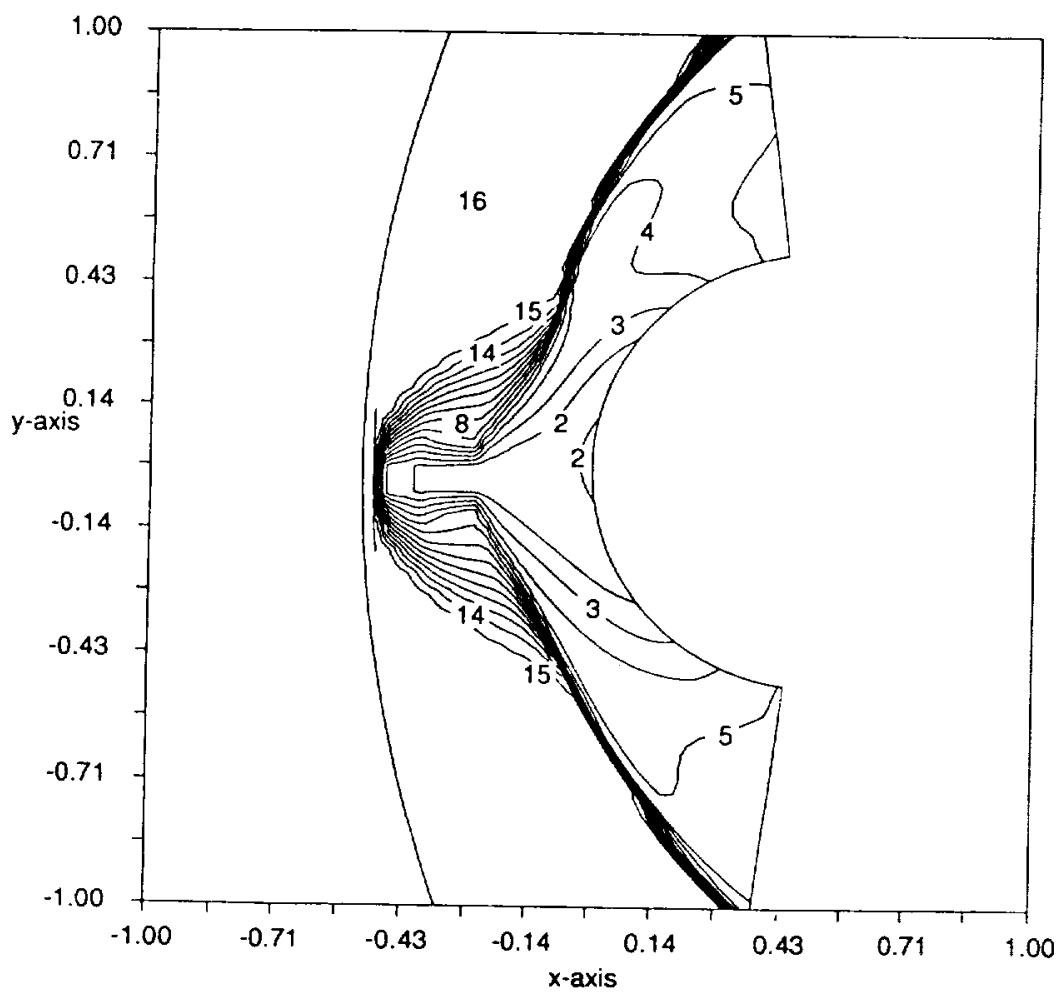

(d)

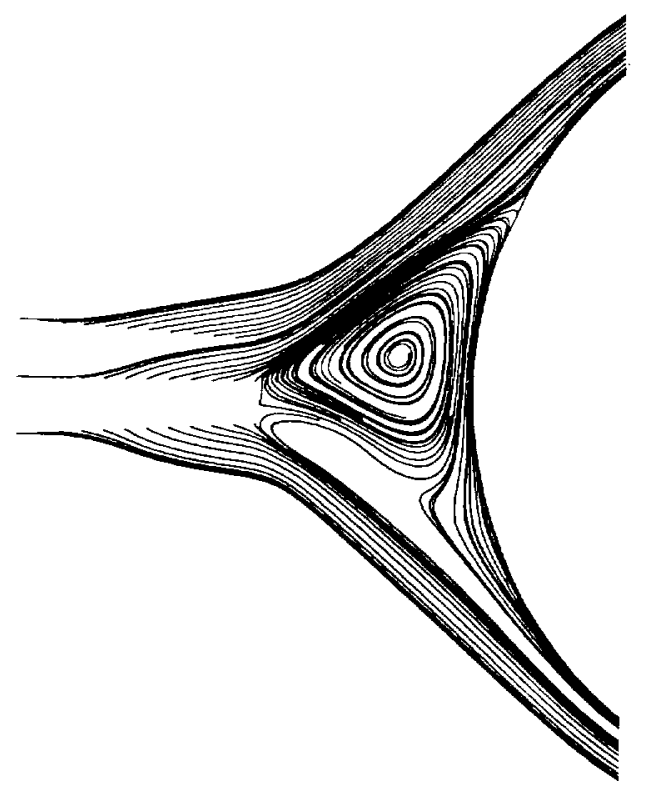

Fig. 14. Mach 6.0 flow over a circular cylinder, Mach Contours: (a) AUSM and (b) Roe FDS. The $45 \times 43$, shock aligned grid is shown in figure (c). The particle traces shown in figure (d) also demonstrate the nonphysical nature of the Roe solution, corresponding to figure (b). 
(a)

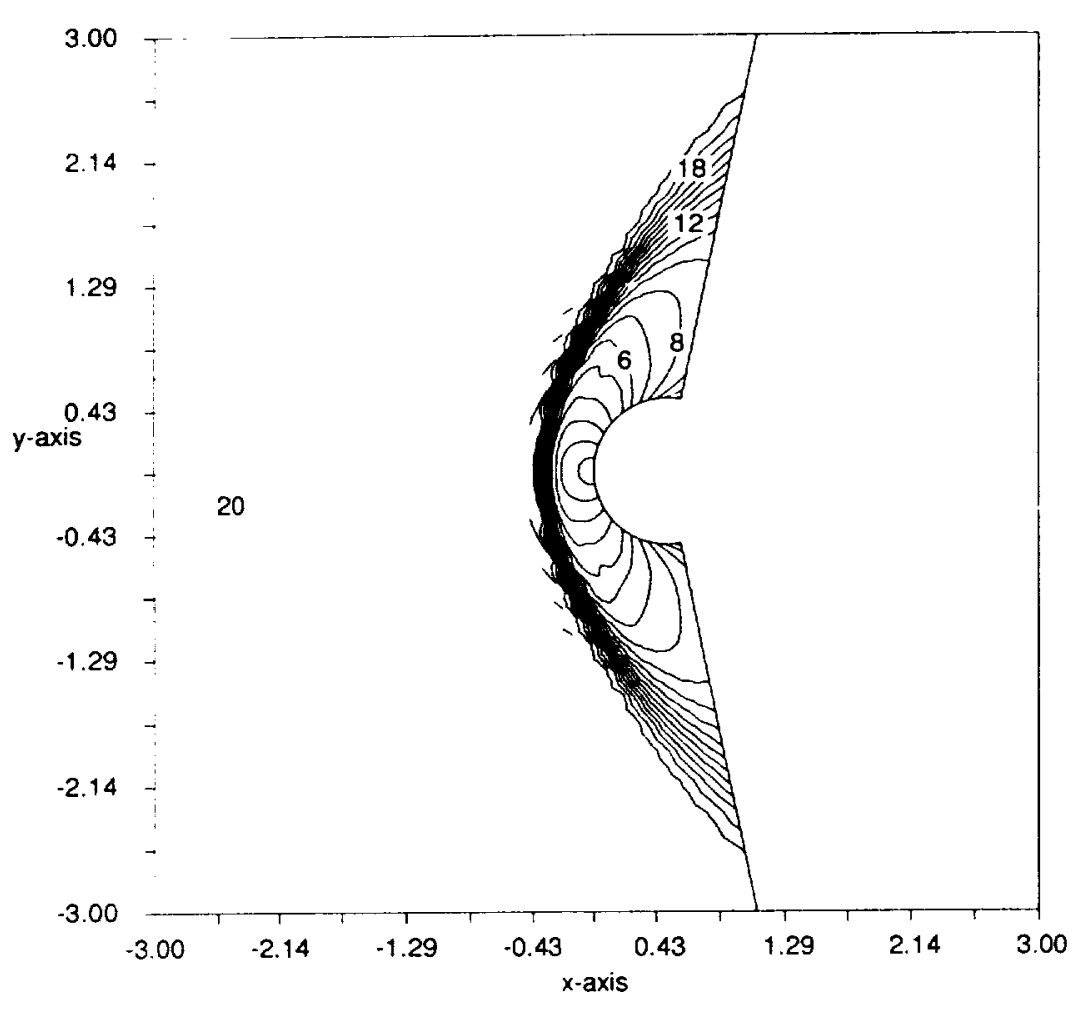

(b)

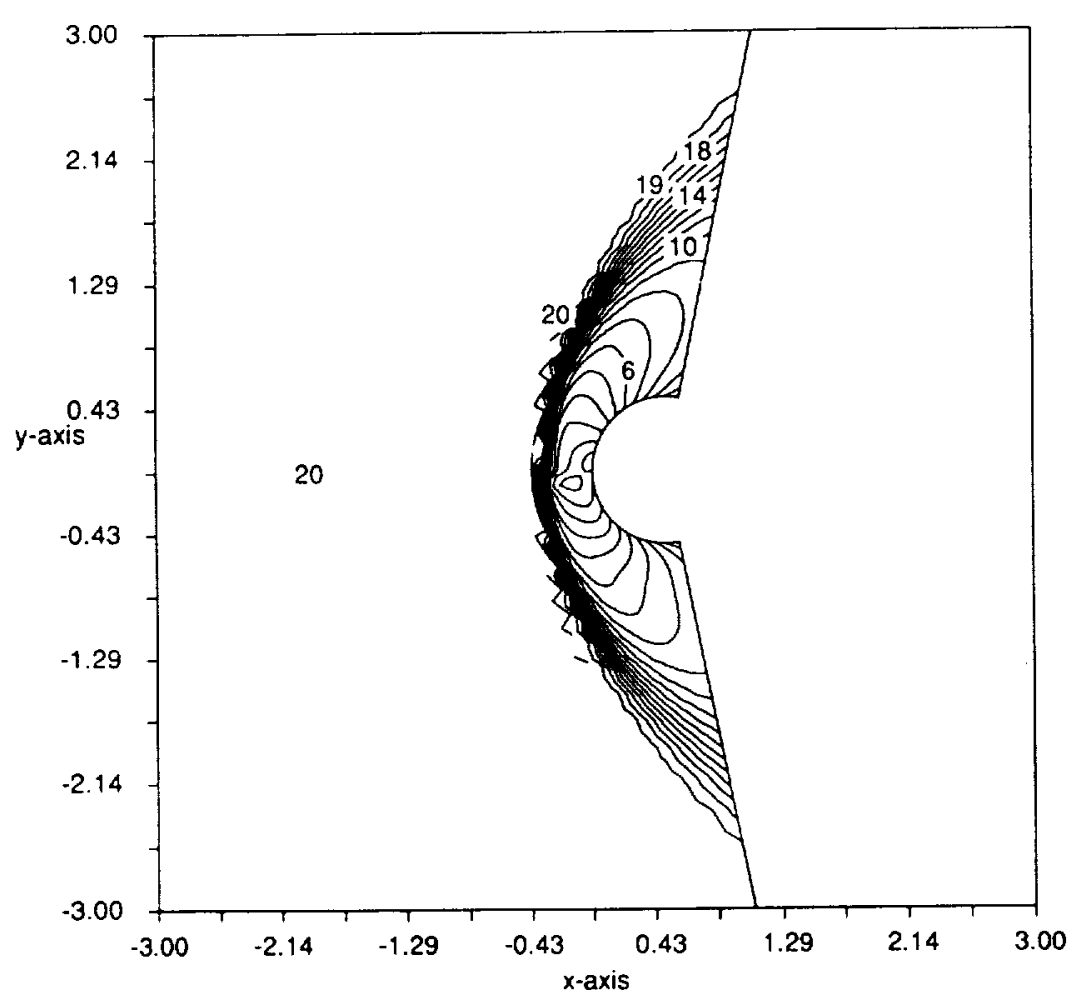

(c)

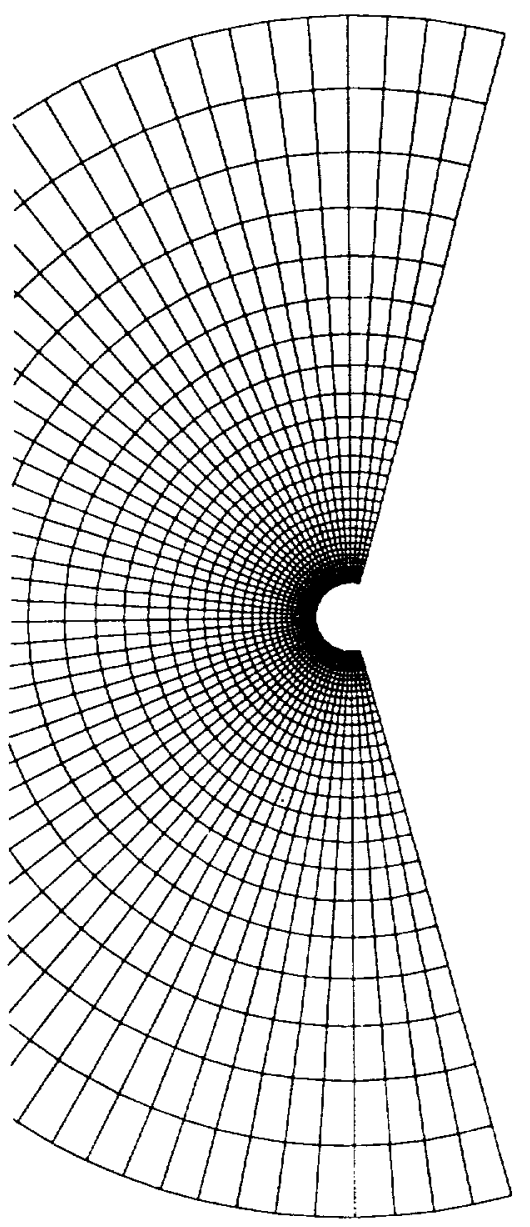

Fig. 15. Mach 4.0 flow over a circular cylinder, Mach Contours: (a) AUSM and (b) Roe FDS. The $57 \times 33$, radially stretched circular grid is shown in figure (c). 
(a)

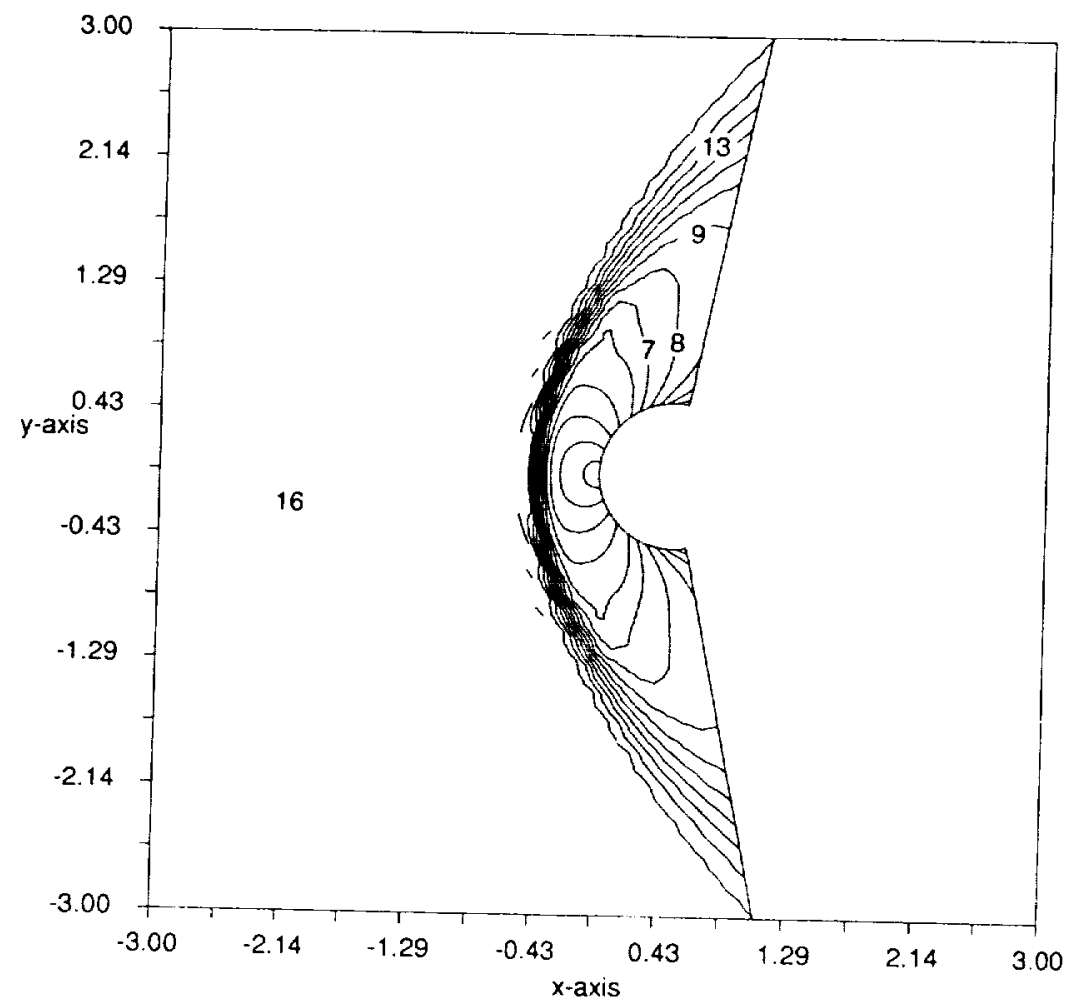

(b)

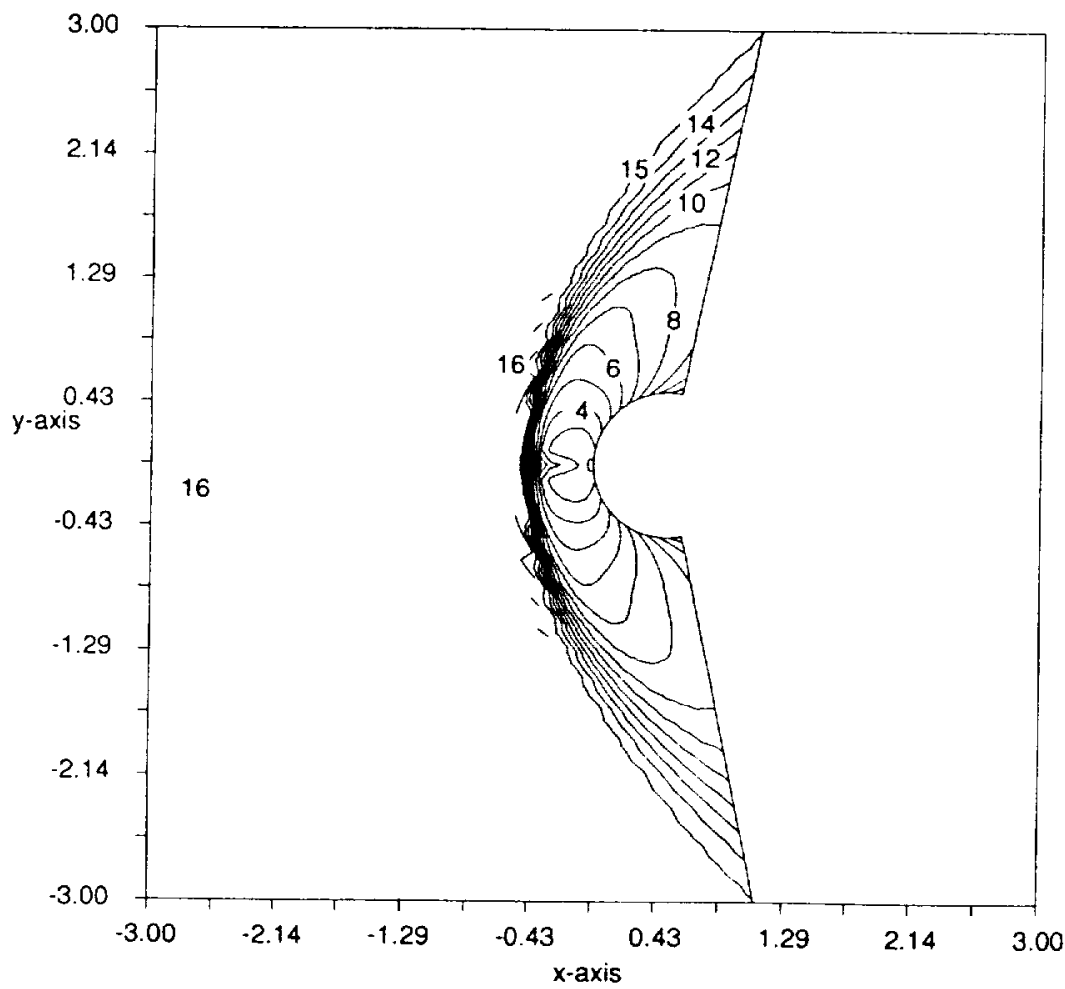

Fig. 16. Mach 3.0 flow over a circular cylinder, Mach Contours: (a) AUSM and (b) Roe FDS. 
(a)

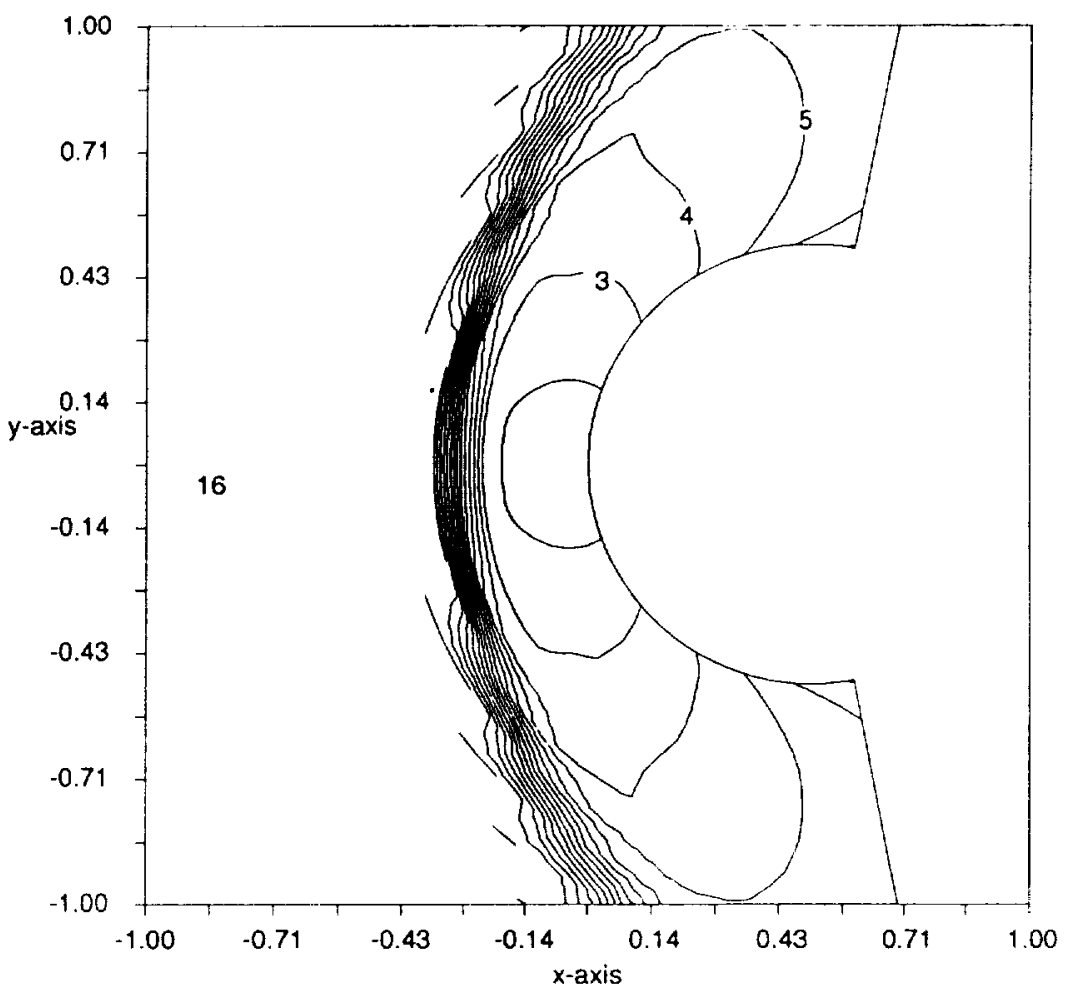

(b)

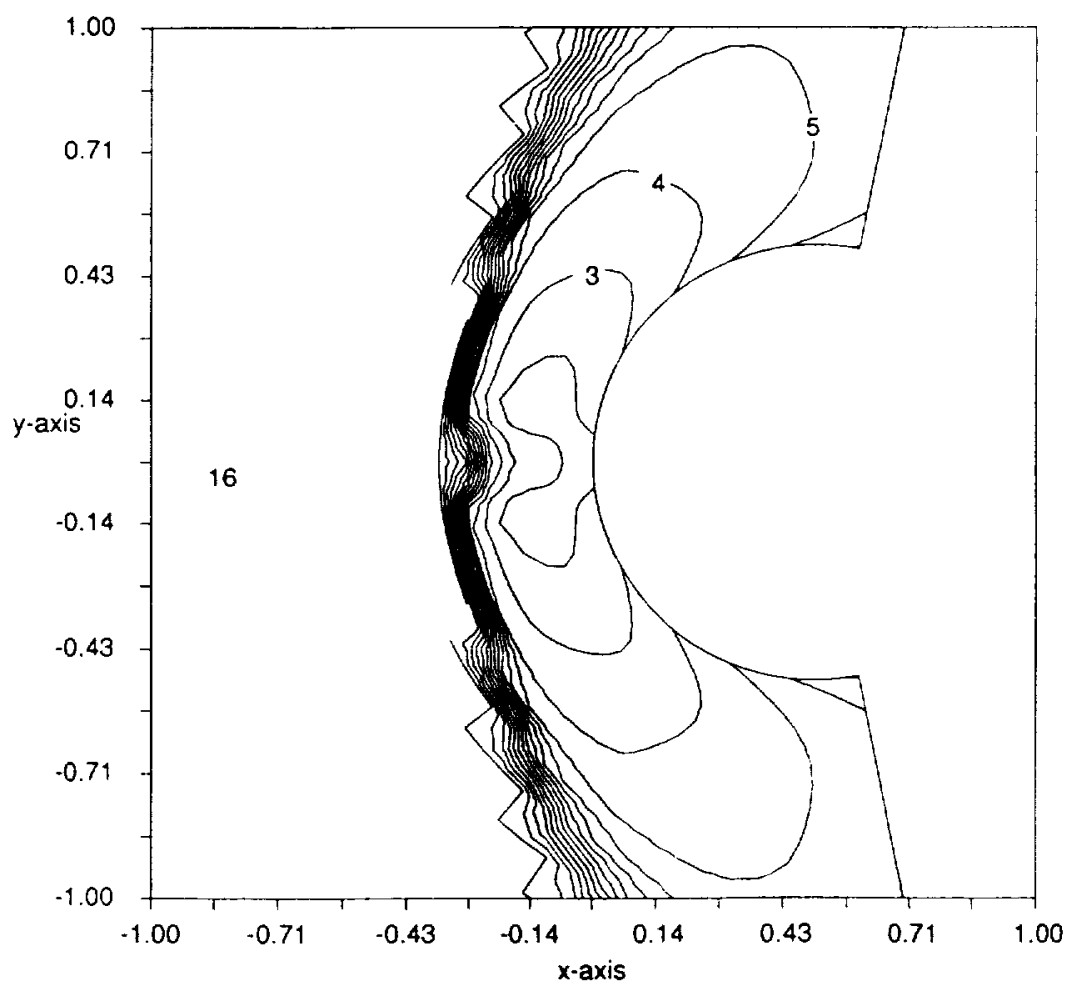

$\begin{array}{cc}1 & 0.0000 \\ 2 & 0.4000 \\ 3 & 0.8000 \\ 4 & 1.2000 \\ 5 & 1.6000 \\ 6 & 2.0000 \\ 7 & 2.4000 \\ 8 & 2.8000 \\ 9 & 3.2000 \\ 10 & 3.6000 \\ 11 & 4.0000 \\ 12 & 4.4000 \\ 13 & 4.8000 \\ 14 & 5.2000 \\ 15 & 5.6000 \\ 16 & 6.0000 \\ 17 & 6.4000 \\ 18 & 6.8000 \\ 19 & 7.2000 \\ 20 & 7.6000\end{array}$

Fig. 17. Mach 6.0 flow over a circular cylinder, Mach Contours: (a) AUSM and (b) Roe FDS. 


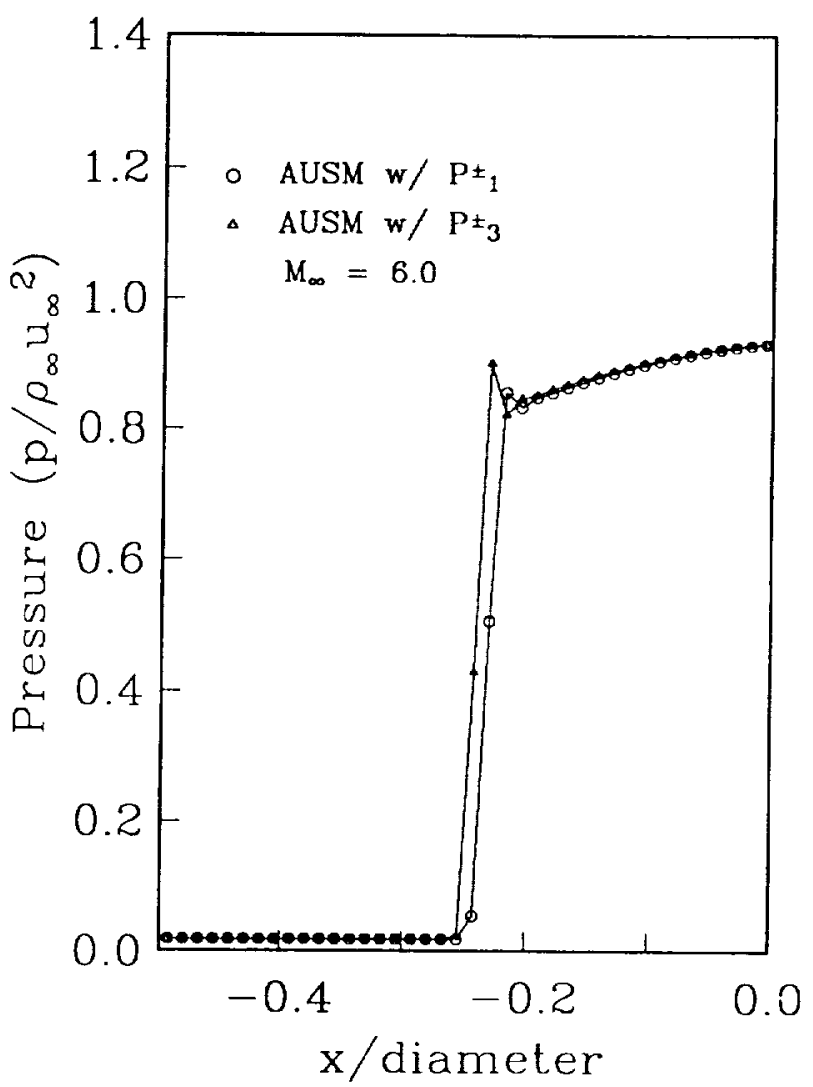

Fig. 18. Effect of different pressure splittings upon the overshoot at a shock wave. 


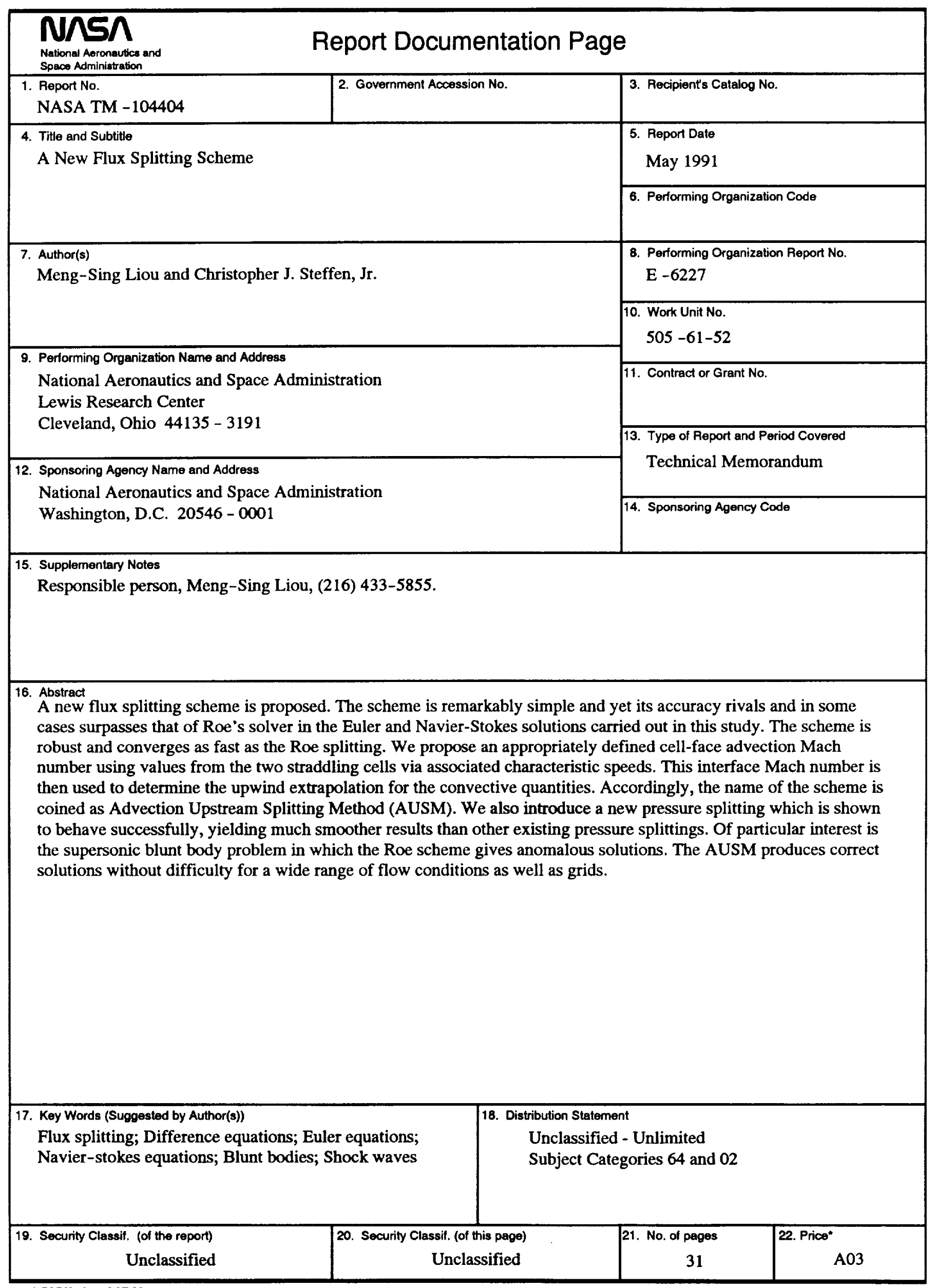




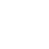


National Aeronautics and

Space Administration

Lewis Research Center

Cleveland, Ohio 44135

Otticial Business

Penalty for Private Use $\$ 300$

Postage and 5 eer rim

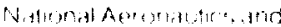

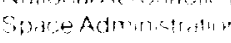

NASA 45 